\title{
Cloud customers service selection scheme based on improved conventional cat swarm optimization
}

\author{
Danlami Gabi ${ }^{1,2} \cdot$ Abdul Samad Ismail $^{3} \cdot$ Anazida Zainal $^{3} \cdot$ Zalmiyah Zakaria $^{3} \cdot$ Ajith Abraham $^{4}$. \\ Nasiru Muhammed Dankolo ${ }^{3}$
}

Received: 15 February 2019/Accepted: 5 March 2020/Published online: 17 March 2020

(C) The Author(s) 2020

\begin{abstract}
With growing demand on resources situated at the cloud datacenters, the need for customers' resource selection techniques becomes paramount in dealing with the concerns of resource inefficiency. Techniques such as metaheuristics are promising than the heuristics, most especially when handling large scheduling request. However, addressing certain limitations attributed to the metaheuristic such as slow convergence speed and imbalance between its local and global search could enable it become even more promising for customers service selection. In this work, we propose a cloud customers service selection scheme called Dynamic Multi-Objective Orthogonal Taguchi-Cat (DMOOTC). In the proposed scheme, avoidance of local entrapment is achieved by not only increasing its convergence speed, but balancing between its local and global search through the incorporation of Taguchi orthogonal approach. To enable the scheme to meet customers' expectations, Pareto dominant strategy is incorporated providing better options for customers in selecting their service preferences. The implementation of our proposed scheme with that of the benchmarked schemes is carried out on CloudSim simulator tool. With two scheduling scenarios under consideration, simulation results show for the first scenario, our proposed DMOOTC scheme provides better service choices with minimum total execution time and cost (with up to $42.87 \%, 35.47 \%, 25.49 \%$ and $38.62 \%, 35.32 \%, 25.56 \%$ reduction) and achieves $21.64 \%, 18.97 \%$ and $13.19 \%$ improvement for the second scenario in terms of execution time compared to that of the benchmarked schemes. Similarly, statistical results based on $95 \%$ confidence interval for the whole scheduling scheme also show that our proposed scheme can be much more reliable than the benchmarked scheme. This is an indication that the proposed DMOOTC can meet customers' expectations while providing guaranteed performance of the whole cloud computing environment.
\end{abstract}

Keywords Cloud computing $\cdot$ Scheduling $\cdot$ Cat swarm optimization $\cdot$ Pareto dominance

Danlami Gabi

danlami@cs.umu.se

Abdul Samad Ismail

abdsamad@utm.my

Anazida Zainal

anazida@utm.my

Zalmiyah Zakaria

zalmiyah@utm.my

Ajith Abraham

ajith.abraham@ieee.org

Nasiru Muhammed Dankolo

ndmuhammad@live.utm.my
Department of Computer Science, Faculty of Science, Kebbi State University of Science and Technology, Aliero, Kebbi State, Nigeria

2 Department of Computing Science, Umea University, Umeå, Sweden

3 Department of Computer Science, School of Computing, Universiti Teknologi Malaysia, Johor, Malaysia

4 Machine Intelligence Research Labs, Scientific Network for Innovation and Research Excellence, Auburn, WA 98071, USA 


\section{Introduction}

Cloud computing is a platform of choice providing distributed resources (e.g., virtual machine, storage and bandwidth) to meet increasing demand of customers. The environment provides cost-effective solution for running business applications through virtualized technologies [1-4]. Services that are made available by cloud environment are affordable using the concept of pay-per-use (PPU) pricing models. Three types of service model are associated with the cloud computing environment: Software as a Service (SaaS), Platform as a Service (PaaS) and Infrastructure as a Service (IaaS) [5]. The cloud customers usually interact remotely with the SaaS layer to run their applications (e.g., datacenter) [5]. The SaaS layer thus functions with the support of PaaS layer. The PaaS layer provides interactive mechanisms between cloud customers and service providers. The PaaS thus allows cost-efficient development and deployment of applications [7, 8]. With the provision of numerous advantages (e.g., deployment of PPU pricing model, maintaining application having the same integrated environment) by the PaaS layer, the cloud customers can now have the means of using same integrated software application development environment $[9,10]$. On the other hand, the IaaS layer is responsible for providing services to the cloud customers in terms of the infrastructures. The cloud customers have no control over managing the infrastructures; rather, they only utilize the available resources situated at the IaaS layer through service request [6]. In another development, the IaaS provides pool of resources of varied types which are leased according to customers' request. This paper provides customers with techniques for optimal mapping of their choice of resources at the IaaS layer to meet their computation need $[7,11,12]$.

At the IaaS layer, virtual machines are heterogeneous. Some requests are said to have a very high demand for virtual machines, while others require more storage $[13,14]$. In most instances, the customers demand resources that have minimum costs of execution. Virtual machines with high processing speed usually incur high processing cost [15-18]. In Amazon EC2 for instance, a cloud customer has the privilege of accessing and controlling set of virtual machines that run inside the datacenter of the service provider, while being charged for a specific time the virtual machine has been allocated. At this instance, the customer satisfaction is measured based on the Quality of Service (QoS) (e.g., execution time, cost and storage) he/or she experiences $[19,20]$. To provide customers of cloud computing resources with better options of selecting their resource preferences, there is need for an optimal resource scheme $[15,16,21,22]$. In recent development, high-level research conducted in cloud computing task and resource scheduling focuses on time and cost models. These models capture customers' QoS experience, where techniques of metaheuristic are exploited in their evaluations. Although the metaheuristic techniques are attributed with certain limitations (e.g., local and global imbalance, local optimality) [14, 23], they have been proven to be more efficient in reducing the complexity of a task scheduling problem when finding an optimum or near-optimal solution [14, 24, 25]. Several researchers [26-31] also focus on investigating relationship between local optimality and slow convergence and proffered solutions to addressing these concerns.

The conventional cat swarm optimization (CSO) is a metaheuristic optimization technique put forward by $\mathrm{Chu}$ and Tsai in 2007. This technique mimics the behavior of natural cat and has relatively proven better in terms of convergence speed than the particle swarm optimization (PSO) [32]. It has both the global and local search (also known as the seeking and tracing mode) and a control variable called the mixed ratio (MR) that determine whether the current position at which the cat is standing is in either seeking or tracing mode. As attributed to most metaheuristic techniques, the CSO suffers slow convergence speed that can lead to its entrapment at the local optima, and global and local imbalance causing instability of the solution. Orthogonal Taguchi approach is a greedybased technique that when applied can increase the convergence speed of the CSO to avoid being trapped at the local optima $[13,20]$. In this paper, we proposed Dynamic Multi-Objective Orthogonal Taguchi-Cat (DMOOTC) scheme. In the proposed scheme, we exploit advantages of the orthogonal Taguchi approach to avoid the DMOOTC from being entrapped at the local optima. To provide customers with choices of service preferences, we incorporated the Pareto dominance strategy in the proposed DMOOTC scheme. The simulation results show that our proposed DMOOTC scheme can provide customers with better service choices compared to the benchmarked schemes.

The contribution of this paper is as follows:

- Development of a multi-objective task scheduling model for cost and computation time.

- An improvement is proposed to the CSO algorithm using the orthogonal Taguchi approach and Pareto dominance.

- Development of a DMOOTC scheme to solve the multiobjective task scheduling model.

The rest of this article is organized as follows: Review on related work is discussed in Sect. 2. Section 3 discusses the CSO technique. Orthogonal Taguchi approach is discussed in Sect. 4. Section 5 provides discussion on Pareto 
dominance strategy. The proposed models are discussed in Sect. 6. Section 7 discusses the proposed DMOOTC scheme. Discussion on simulation results is provided in Sect. 8. Section 9 discusses the performance metrics used in the evaluation of the schemes. Discussion on the simulation results is provided in Sect. 10. Section 11 provides discussion on the statistical results, and finally, Sect. 12 concludes the paper.

\section{Related works}

Task scheduling is one of the most research areas in cloud computing. Researchers have shown that scheduling strategies such as heuristic and metaheuristic can be promising when applied to deal with scheduling problems in cloud datacenters. Metaheuristics such as particle swarm optimization (PSO), genetic algorithm (GA), ant colony optimization (ACO) and cat swarm optimization (CSO) are few examples of metaheuristic techniques that can handle large scheduling problems. Their improvement with trajectory-based (e.g., simulated annealing (SA)) and greedybased techniques such as the orthogonal Taguchi approaches can further improve their performances toward providing more efficient solutions [33-35]. Few among researchers that exploit these advantages are discussed in the following:

In Wei et al. [36], a Compounded Local Mobile Cloud Architecture (LMCpri) with dynamic priority queue is proposed to solve a multi-objective scheduling problem. In their proposed approach, a priority-based positioning technique based on auction processing is incorporated to store jobs upon arrival from the cloud customers. Then, a Non-Static Genetic Algorithm II (NSGA-II) is introduced for scheduling tasks on resources to achieve minimum processing time and decrease the request cost. According to the researchers, simulation results show their proposed algorithm can provide better performance than PSO and sequential scheduling algorithms in terms of minimum total execution time and cost. However, improvement in their proposed method is still possible since mutation process exhibited by the GA can lead to local trapping due to slow convergence speed. In another development, Liu et al. [37] proposed a Single Site Virtual Machine Provisioning (SSVP) approach and ActGreedy to minimize task execution time and monetary cost. In their proposed approach, a single-site initialization module is used to ensure virtual machine provisioning and multisite data transfer. At the instance of task execution, a virtual machine is not allowed to restart for the execution of any continuous activities on the site due to the fact that they are grouped and scheduled as a fragment. According to the researchers, simulation results show their developed SSVP can generate better VM-provisioning plan for customers to achieve minimum task execution time and monetary cost compared to the benchmarked techniques.

In Zuo et al. [14], a Multi-Objective Ant Colony Optimization (MOACO) algorithm is proposed. Their objective is to minimize the makespan time and budgetary cost. A cost model that reflects the relationship between customers' resource and budgetary cost is introduced in evaluating the efficiency of their proposed algorithms. According to the researchers, simulation results show their proposed algorithm has achieved minimum execution cost compared to that of the benchmarked algorithms. However, the updating process of pheromone exhibited by the ants along the path can lead to local trapping. Duan et al. [38] in their part proposed a communication and storage-aware multi-objective task scheduling algorithm, which is based on sequential cooperative game. Their goal is to optimize the execution time and economic cost. In their proposed approach, the individual players are considered behaving selfishly. The global knowledge of all players is computed by ordering each customer's task in decreasing order. The simulation results show their proposed algorithm can achieve better solution in terms of makespan, cost, systemlevel efficiency and fairness in less execution time compared to Grid-Min-Min, Grid-Max-Min and GridSuffrage.

In Verma and Kaushal [39], a Hybrid Particle Swarm Optimization (HPSO) algorithm is proposed. Their objective is to provide resources that can guarantee customers with minimum execution time and processing cost under deadline and budget constraint. In their studies, trade-off values are adopted to provide customers with the choices of selecting their service preferences. According to the researchers, simulation results shows their proposed HPSO scheduling approach can reduce execution time and execution cost as compared to the Non-Static Genetic Algorithm II (NSGA-II), Multi-Objective Particle Swarm Optimization (MOPSO) and $\varepsilon$-Fuzzy Dominance sortbased Discrete Particle Swarm Optimization ( $\varepsilon$-FDPSO) scheduling algorithm. However, the global optimization process exhibited by the PSO may not always guarantee the required optimum solution. Panda and Jana [34] in their part proposed a Multi-Objective Task Scheduling (MOTS) optimization algorithm for heterogeneous multi-cloud environment. Their goal is to minimize both makespan time and total execution cost. In their method, two phases of task scheduling processes were adopted. The first phase goes through normalization process to scale values between 0 and 1 . In the second phase, the normalization process is performed by dividing the Expected Time to Compute (ETC) matrix and cost matrix element into their corresponding maximum values. As put forward by the researchers, simulation results show their proposed method 
has outperformed the two benchmarked algorithms in achieving minimum execution time, minimum total cost of execution and improves the average cloud utilization. Ramezani et al. [15] introduced a Multi-Objective Particle Swarm Optimization (MOPSO) algorithm to provide customers with better service choices to deal with the challenges of high computation time and cloud performance. As stated by the researchers, their simulation results show they can achieve an optimal solution in a reasonable amount of time. However, incorporating a novel technique can further improve their solution in terms of generating trade-offs for consumers' service preferences during task scheduling.

Gabi et al. [33] put forward an Orthogonal TaguchiBased Cat Swarm Optimization (OTB-CSO) algorithm to improve the performance of cloud environment. The researchers exploited the advantages of Taguchi method to achieve task mapping on appropriate virtual machines. The simulation results according to the researchers show their proposed algorithm can reduce makespan compared to Min-Max, Hybrid Particle Swarm Optimization with Simulated Annealing (HPSO-SA) and Particle Swarm Optimization with Linear Descending Inertia Weight (PSO-LDIW). However, the improvement in their OTBCSO to handle a multi-objective optimization problem is required to meet customers' expectation. Liu et al. [40] dwelt on Improved Min-Min algorithm for cloud computing environment. Their objective is to achieve QoS, dynamic priority model and minimum cost of service delivery to customers. In their scheduling process, static priority rule and dynamic changing factors are used for providing the scheduling of higher priority tasks first. The results of their simulation as indicated by the researchers show their proposed algorithm can increase resource utilization, ensure longer task is executed at reasonable time and meet customers' QoS requirement compared to the benchmarked algorithm. In another development, Beegom and Rajasree [41] in their part put forward a new variant of continuous Particle Swarm Optimization (PSO) algorithm called the Integer-PSO. The Integer-PSO adopts Pareto optimality using a weighted sum approach. Their goal is to minimize the makespan and execution cost. In their scheduling process, a model as a constraint biobjective optimization for the makespan and cost is developed. The efficiency of their proposed algorithm is tested using the developed model. Simulation results as shown by the researchers indicate that their proposed Integer-PSO has outperformed the Smallest Position Value (SPV) rulebased PSO technique in terms of achieving minimum makespan and execution cost.

To ensure effective scheduling on heterogeneous virtual machines and reduce task execution time, Akbari and Rashidi [42] proposed a Multi-Objective Scheduling
Cuckoo Optimization Algorithm (MOSCOA). In their proposed approach, each cuckoo is used as a scheduling solution. As tasks are placed in order of their arrivals, the cuckoo technique in turn does the mapping to the most appropriate virtual machines as it enables the movement of tasks toward the global optima region using a target immigration operator. The technique was later evaluated using large number of random graphs and real-world application. The simulation results show their proposed MOSCOA algorithm is much more superior in terms of performance compared to their previously proposed task scheduling algorithm. Voicu et al. [43] introduced MultiObjective and Multi-Constrained (MOMC) task scheduling algorithm for scheduling tasks in Hadoop system. Their objective is to minimize deadline and budget. The simulation results according to the researchers show their proposed MOMC method can provide better performance in Hadoop system.

In Bilgaiyan et al. [44], a Multi-Objective Cat Swarm Optimization (MOCSO) algorithm is proposed. Their goal is to improve the performance of cloud environment in terms of minimum execution cost, makespan and CPU idle time. In their task scheduling process using the proposed MOCSO, a control variable known as the mixed ratio is used to decide the best virtual machines to assign tasks. The experimental results according to the researchers show their proposed MOCSO algorithm can achieve minimum execution cost, makespan time and CPU idle time than Multi-Objective Particle Swarm Optimization. On the other hand, Xu et al. [45] in their part proposed a Multi-Objective Genetic Optimization Algorithm (MOGA). Their goal is to minimize the average completion time, total completion time and ensure load balancing on virtual machines. In their scheduling process, large tasks are divided into multiple sub-tasks using the chromosomes encoding. Each chromosome length signifies length of the sub-tasks with smaller tasks mapped on virtual machines. To determine the performance of their proposed MOGA, three different fitness functions models were designed to evaluate the fitness of each chromosome according to their objectives. The simulation results presented by the researchers show their proposed MOGA can achieve the minimized average completion time, total completion time and ensure load balancing on virtual machines with faster convergence than the benchmarked single-objective genetic algorithms. In their part, Milani and Navin [46] proposed a multi-objective scheduling algorithm based on PSO technique. Their objective is to minimize the total execution time, average waiting time and number of missed tasks. The researchers exploit the PSO technique to propose a scheduling approach that can allocate tasks on the best virtual machines. To investigate how efficient is their solution, a fitness function model is developed. The experimental 
results as put forward by the researchers show their proposed algorithm can achieve minimum execution time, waiting time and missed tasks compared to First Come First Served (FCFS), Shortest Process Next (SPN) and Highest Response Ratio Next (HRRN).

Jena [47] proposed a multi-objective Two-State PSO (TSPSO) algorithm. Their aim is to reduce the energy consumption and makespan at the cloud datacenters. In their scheduling method, selection of best virtual machine is introduced using the nondominance strategies. As the tasks are scheduled across virtual machines, the two-stage PSO generates two fitness functions and are compared to determine the solution that is nondominant. The nondominant solution is then chosen to represent the optimum solution of the task scheduling process. According to the researchers, the simulation results show their proposed TSPSO algorithm can minimize the energy and makespan compared to Best Resource Selection (BRS) and Random Scheduling Algorithm (RSA). In Khajehvand et al. [48], the researchers introduced a hybrid First-Fit Cost-Time Trade-Off (FCTT) and Workflow Planning Cost-Based (WPC) model to minimize the runtime and execution cost of scheduled tasks on virtual machines. In their scheduling process, large task is divided into sub-tasks which are sorted in nonincreasing manner. In their proposed algorithms, a bottom-up traversal technique is later incorporated to assign each sub-task a rank. The child sub-tasks are first allocated virtual machines for their execution. The parent tasks are later executed only when the child tasks completed their execution on the virtual machines. According to the researchers, their simulation results show the proposed FCTT can reduce task runtime and execution cost compared to MOGA and Best Effort (BE) algorithms. However, task updates method exhibited by their proposed WPC technique can lead to longer execution time since the techniques. Although the metaheuristics are promising than the heuristic techniques, their improvements using a trajectory-based technique like the simulated annealing as well as the greedy-based techniques like the orthogonal Taguchi approach can enable the metaheuristics to become a potential solution in solving a multi-objective task scheduling problem in cloud computing environment. Therefore, this paper addresses the concern of customers service selection strategy using an improved metaheuristic algorithm to meet customers' QoS expectations with a focus on multi-objective task scheduling.

\section{Cat swarm optimization}

Chu and Tsai [32] proposed the CSO technique. The technique mimics the common behavior of natural cat. The CSO technique has two modes of operation: resting (seeking) and chasing (tracing) mode. The two modes are also referred to as the global and local search. A control factor within CSO known as the mixed ratio (MR) is used to determine the current position of the cat. The cat position also signifies solution (fitness) set. The velocity of the cat is associated with a dimension and a fitness value. As the cat progresses closer to the solution (fitness), it updates itself each time with better results at the memory until all the cats achieve the best solution (fitness) [20, 32, 49]. The following sections explain the seeking and tracing modes $[20,49,50]$.

\subsection{Seeking mode}

The seeking mode is known as the global search process of the CSO technique [50]. Algorithm 1 shows the pseudocode for the seeking mode $[49,50]$.

\begin{tabular}{|c|c|}
\hline \multicolumn{2}{|c|}{ Algorithm 1: Seeking Mode Process } \\
\hline & $\begin{array}{l}\text { Generate } \mathrm{Y} \text { copies of cats k-th, i.e } Z_{d q} \text { where, }(1 \leq q \leq Y) \text { and }(1 \leq d \leq D) \text { where, } D \text {. } \\
\text { is the overall dimension of the problem and } \mathrm{Y} \text { is the seeking memory pool. }\end{array}$ \\
\hline 2. & $\begin{array}{l}\text { Change at random the dimensions of the cats as per CDC by applying mutation } \\
\text { operator to } Z_{d q} \text {. Where } \mathrm{CDC} \text { is the count dimension to change }\end{array}$ \\
\hline 3. & Determine the fitness values of all changed cats. \\
\hline 4. & Discover the best cats (non-dominant) based on their fitness values. \\
\hline 5 . & Replace the position of the k-th cat after picking a cat at random from $\mathrm{Y}$. \\
\hline
\end{tabular}

performance of the algorithm depends on its update process.

Form the literature reviewed so far, high complexity, slow convergence and imbalance between global and local search are some of the drawbacks of the metaheuristic

\subsection{Tracing mode}

The tracing mode corresponds to the local search process of the CSO. The pseudocode for the tracing mode is shown in Algorithm 2 [20, 49, 50]. 


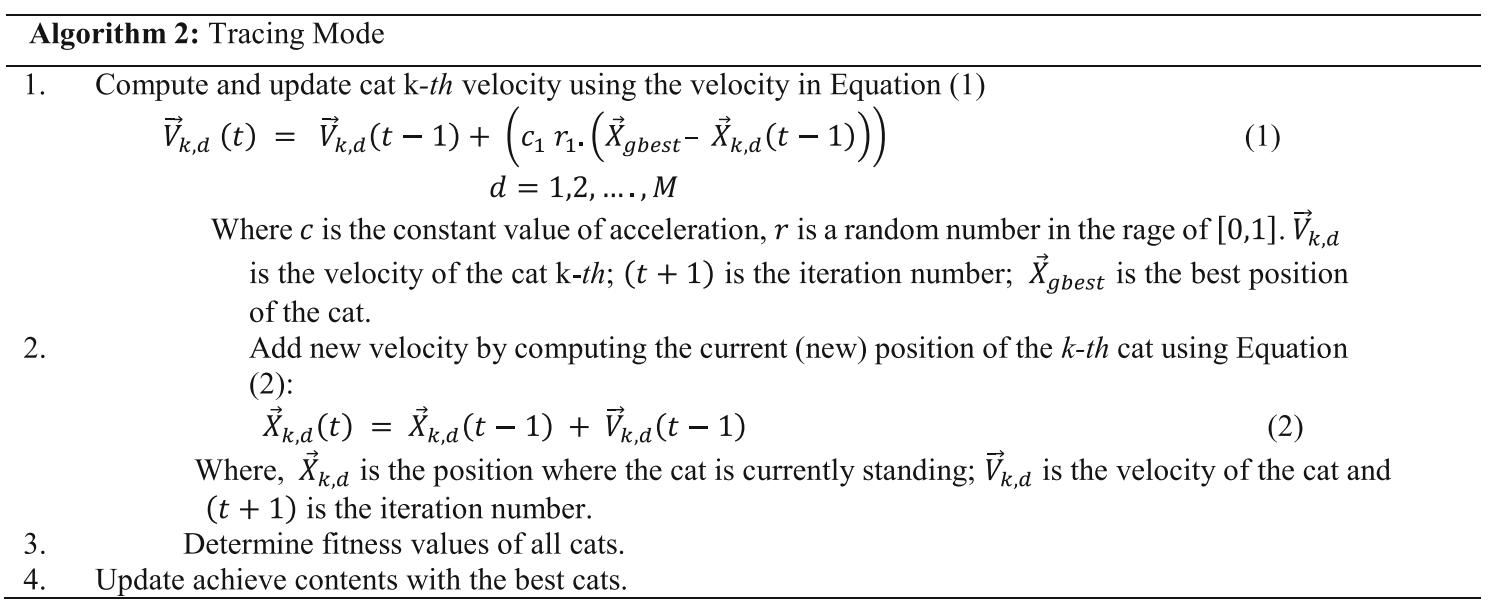

\subsection{The need for cat swarm optimization improvement}

To provide efficient scheduling in cloud datacenters with the goal of meeting customers' expectations, the CSO then needs to be improved. However, global search of the CSO does not always assure superior solutions when the search space increases. The number of cats that always move into the global search mode of the CSO always exceeds that of the local search. Thus, its convergence toward a stable solution becomes difficult, leading to its entrapment at the local optima [32]. On the other hand, for each iteration, the global and local search modes exhibited by the CSO are independently carried out. These also cause its velocity and position update to perform similar process. This can lead to high computation time during task scheduling on cloud computing environment. In another concern, the CSO can only handle a single-objective optimization problem. Imbalance between global and local search of the CSO becomes another challenge. Hence, there is a need for improving CSO to make it efficient for service provisioning in cloud computing [33].

\section{Taguchi orthogonal array}

Taguchi method is a greedy approach put forward by Genichi Taguchi [51]. The Taguchi method uses an orthogonal array (OA) matrix representation for its experiment. It involves the study of a large number of design variables to achieve efficient results using few numbers of simulation runs [52]. According to Taguchi, for any twolevel orthogonal array (2OA) with $Z$ factors where $Z$ represents the number of designed factors, each factor will be based on two levels. Taguchi formulated a general symbol shown in Eq. (3) for the establishment of an OA with $n$ levels of $Z$ factors [53, 54]:
$L_{n}\left(2^{n-1}\right)$,

where $n-1$ represents the number of columns in two-level orthogonal array; $n=2 k$ is the number of experiments corresponding to the $n$ rows and columns; 2 represents the number of levels required for each factor $Z$; and $k$ is a positive integer $(k>1)$. The matrix in Table 1 shows the values in the column are mutually orthogonal. According to Taguchi, for any pair of columns, the combinations of all factors at each level occur at an equal number of times. As described in [45], to allocate six factors each with two levels " $L_{8}\left(2^{6}\right)$," only six columns are needed for the run of the experiment. Hence, $L_{8}\left(2^{7}\right)$ orthogonal seems sufficient, since there are seven columns. The $L_{8}$ is an indication that eight experimental runs will be conducted by studying seven variables at two levels. The value "7" represents the dimension of the problem. The main objective of adopting the Taguchi approach is to find an optimal solution in a reasonable amount of time $[51,55]$. Detail about the Taguchi method can be found in [33].

Here, the value 1 at each column represents the first set of factors to be considered for the experiment, while the value 2 represents the second set of experiments.

Table $1 \quad L_{8}\left(2^{7}\right)$ OA [27]

\begin{tabular}{|c|c|c|c|c|c|c|c|}
\hline \multirow[t]{3}{*}{ Experiment number } & \multicolumn{6}{|c|}{ Factors } & \\
\hline & A & B & $\mathrm{C}$ & $\mathrm{D}$ & E & $\mathrm{F}$ & $C$ \\
\hline & \multicolumn{6}{|c|}{ Column numbers } & \\
\hline 1 & 1 & 1 & 1 & 1 & 1 & 1 & 1 \\
\hline 2 & 1 & 1 & 1 & 2 & 2 & 2 & 2 \\
\hline 3 & 1 & 2 & 2 & 1 & 1 & 2 & 2 \\
\hline 4 & 1 & 2 & 2 & 2 & 2 & 1 & 1 \\
\hline 5 & 2 & 1 & 2 & 1 & 2 & 1 & 2 \\
\hline 6 & 2 & 1 & 2 & 2 & 1 & 2 & 1 \\
\hline 7 & 2 & 2 & 1 & 1 & 2 & 2 & 1 \\
\hline 8 & 2 & 2 & 1 & 2 & 1 & 1 & 2 \\
\hline
\end{tabular}




\subsection{The Taguchi optimization algorithm}

The Taguchi method can serve as a better approach in reducing the execution time of a task when used for solving task scheduling problem in cloud computing. In cloud task scheduling, the total cost of execution is mostly influenced by amount of time tasks are executed on a virtual machine. Hence, incorporation of Taguchi optimization algorithm into a conventional CSO can be a potential solution to achieve the desired results. The pseudocode for the Taguchi optimization algorithm is shown in Algorithm 3 $[54,55]$. the cloud customers' attention can be drawn to the trade-off points $P^{*}$ known as the Pareto front, where customers are allowed to select their service preferences in terms of virtual machines that can provide them with the minimum execution time and cost [48, 58]. At this instance, the customers are left to optimize their service preferences by selecting the best trade-offs $[57,58]$. The main goal of this research is to make sure that the Pareto optimal set is discovered in a minimum amount of time for all the tasks scheduled on virtual machines using our proposed technique. This study is based on the following definitions in solving the multi-objective task scheduling problem.

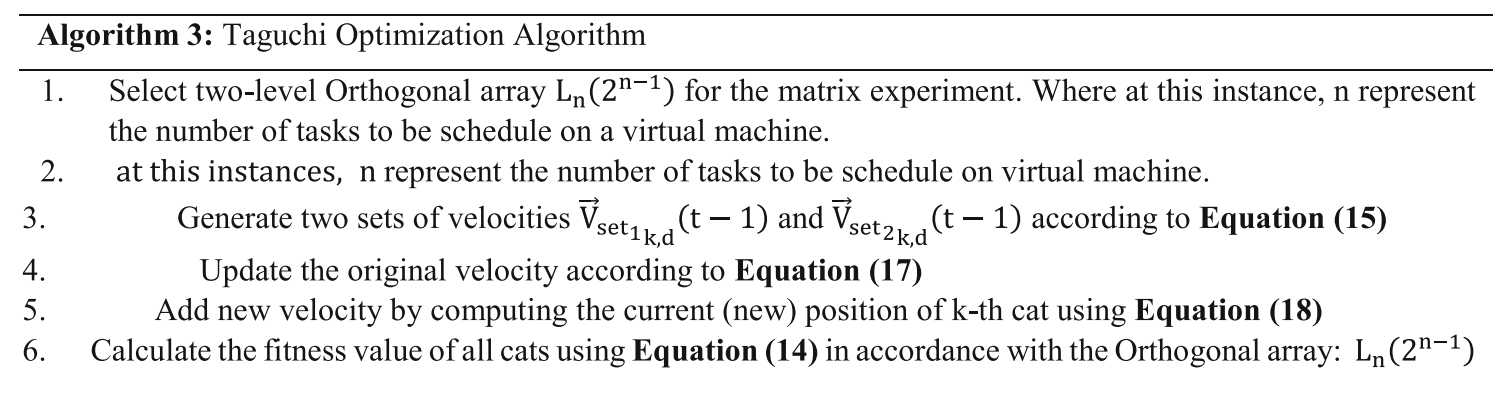

\section{Pareto dominance strategy}

For any task scheduling, the chances of locating an optimal solution that can meet customers' expectations in terms of minimum execution time and cost are becoming harder in a large search space like the cloud computing environment [56]. Due to multi-criteria requirements associated with customers' request, the concept of optimality needs to be achieved. Multi-objective optimization approach is a potential solution to solving this kind of problem. It is characterized with trade-off factors, where each trade-off solution corresponds to a specific order of importance of the objectives [18]. Currently, the Pareto optimization strategy is the most widely adopted strategy for solving several multi-objective problems. Individual objectives can be combined using Pareto dominance strategy to achieve their Pareto front [50]. The Pareto optimal solution that represented best possible among these objectives without worsening another objective is chosen as the best candidate solution $[24,39,53]$.

Task scheduling to meet the expectation of customers involves dealing with multi-objective problems. The Pareto optimization approach can be adopted to provide customers as many nondominant solutions as possible by allowing a set of trade-offs in terms of execution time and execution cost $[37,57]$. Although study shows that the actual customers' service preferences are quite difficult to predict,
Definition 1.1 Multi-objective Optimization problem.

A typical multi-objective optimization problem can be expressed as a minimization of a $K$ components of a vector function $f_{i}$ in the form $[15,49]$ :

$\operatorname{Min} \vec{f}(\vec{x})=\left[f_{1}(\vec{x}), f_{2}(\vec{x}), f_{3}(\vec{x}), \ldots, f_{k}(\vec{x})\right]$,

where $\vec{x} \forall \vec{x}=\left\{x_{1}, \ldots,\right\}$ represents the vector of decision variable such that $f_{i}: R^{n} \rightarrow R, i=\{1,2, \ldots, k\}$ are the objective functions in a universe $U \cdot \vec{f}(\vec{x})$ is the multiobjective function.

\section{Definition 1.2 Pareto Dominance}

In Pareto dominance, two different participants can be judged on how good they are in terms of their performances. Given two candidate solutions $\overrightarrow{x_{1}}$ and $\overrightarrow{x_{2}}$ from $U$, vector $\overrightarrow{x_{1}}$ is said to dominate vector $\overrightarrow{x_{2}}$ (denoted as $\left(\overrightarrow{\left.x_{1}\right)} \prec \overrightarrow{\left(x_{2}\right.}\right)$ ) if and only if:

$f_{i}\left(\overrightarrow{\left.x_{1}\right)} \leq f_{i}\left(\overrightarrow{x_{2}}\right), \quad \forall i \in\{1, \ldots, k\}\right.$

$f_{i}\left(\overrightarrow{\left.x_{1}\right)}<f_{i}\left(\overrightarrow{x_{2}}\right), \quad \exists i \in\{1, \ldots, k\}\right.$

Equation (5) shows that $\overrightarrow{x_{1}}$ dominates $\overrightarrow{x_{2}}$ in all objectives, while Eq. (6) shows $\overrightarrow{x_{1}}$ dominates for at least one of the objectives [7].

\section{Definition 1.3 Pareto Optimal}


If for all means $\overrightarrow{x_{1}}$ is not dominated by any other solution, $\overrightarrow{x_{1}}$ is then considered to be the nondominant (Pareto optimal) solution.

\section{Definition 1.4 Pareto Optimal set}

Set of all solutions $\overrightarrow{x_{1}} \in U$ correspond to the Pareto optimal.

\section{Definition 1.5 Pareto Front $\left(\mathrm{P}^{*}\right)$}

The fitness value of the solution is called trade-off or $\mathrm{P}^{*}$ if and only if Eq. (7) holds. This is the optimal solution of a multi-objective optimization problem that is comprised of a set of solutions:

$f\left(\overrightarrow{\left.x_{1}\right)} \prec f\left(\overrightarrow{x_{2}}\right), \neg \exists \overrightarrow{x_{1}} \in U\right.$

where $\neg$ is true if $\overrightarrow{x_{1}} \in U ; \exists$ means there exist $\overrightarrow{x_{1}} \in U ; \in$ represents an element of $U$; and $U$ is the universal set.

The framework that describes the scheduling process using the developed scheme is discussed in the following.

\section{The system scheme}

Cloud computing consists of several datacenters that are usually managed by the cloud service providers. For any cloud, virtual machines are dynamically created and deployed in datacenters based on task availabilities. These virtual machines are heterogeneous in nature, having different characteristics in terms of memory and sizes. Our assumption is that one datacenter is not sufficiently enough to handle our task scheduling problem. Therefore, two datacenters each with 20 virtual machines are sufficiently enough for our task scheduling problem. Our proposed system scheme is illustrated in Fig. 1. The scheme integrates Pareto optimization strategy for generating a set of tradeoffs in finding the best schedule that can minimize the execution time and cost. Three modules (customers, Pareto generator and scheduler) are defined in the proposed scheme, where each of the modules consists of sub-modules that carry out the scheduling process. The proposed scheduling scheme adopts the DMOOTC algorithm to make the scheduling decisions. The global and local resource managers within the scheme work together with the scheduler to achieve near-optimal solution. In the scheduling process, customers submit their request based on certain resource requirements. The task manager is responsible for estimating the amount of resources to execute the customer's requests. On the other hand, the local resource manager is responsible for monitoring and managing local virtual nodes and to obtain information about processing elements and memory information and bandwidth which are later submitted to the global manager for subsequent forwarding to the scheduler. The Pareto generator then generates a set of trade-offs according to the customer's requirement which are presented to the customer to select his/or her choice. The customer opts for his/or her choice of service preference (i.e., the best virtual machine), and the process continues with the scheduler. The DMOOTC task scheduling algorithm then judges whether available resource has met requirement of the task in terms of time and cost. Then, the proposed DMOOTC algorithm dispatches the customer's task on the chosen virtual resources. The main contribution of this work lies in that the proposed DMOOTC task scheduling algorithm can address uncertainties by allowing customers to realize better performance to cost and time ratio in cloud computing environment.

\subsection{Problem description}

The problem is first represented by using a set of independent tasks waiting to be scheduled on sequence of heterogeneous virtual machines. $V=\left\{v_{k} m \geq k \geq 1\right\}$ is a set of virtual machines, where $m$ is the number of virtual machines. $T=\left\{t_{i} n \geq i \geq 1\right\}$ represents the tasks' groups, and $n$ is the overall number of tasks [37]. Our goal is to dynamically assign each task $t_{i} \forall i=\{1,2, \ldots, n\}$ as customers' requests on appropriate virtual machines $v_{k} \forall k=$ $\{1,2, \ldots, m\}$ in order to determine the timing and execution cost of the tasks. We assume the following in our scheduling problem: (1) Two datacenters are used for the tasks scheduling; (2) the datacenters are said to belong to the same service provider; (3) tasks are assigned to virtual machines dynamically where the total number of all possible schedules is $(n !)^{m}$ for the problem with $n$ number of tasks and $m$ number of virtual machines; (4) preemptive scheduling allocation is not allowed; and (5) the cost of using a virtual machine for a time quantum varies from one to the other. By adopting the Expected Time to Compute (ETC) matrix as shown in Eq. (8), our goal is to dynamically assign each virtual machine $v_{k}$ with the right computing capacity to appropriate customers' request in order to find the optimum value of the total execution time and the total execution cost $[34,53]$ :

ETC $=\left[\begin{array}{cccccc}t_{1} v_{1} & t_{1} v_{2} & \cdot & . & . & t_{1} v_{k} \\ t_{2} v_{1} & \cdot & \cdot & . & . & . \\ \cdot & \cdot & \cdot & . & . & . \\ \cdot & \cdot & \cdot & \cdot & \cdot & \cdot \\ t_{n} v_{1} & \cdot & \cdot & \cdot & \cdot & t_{n} v_{k}\end{array}\right]$

\subsection{The proposed multi-objective task scheduling model}

Our proposed multi-objective time and cost model is formulated from the problem description. The model reflects 


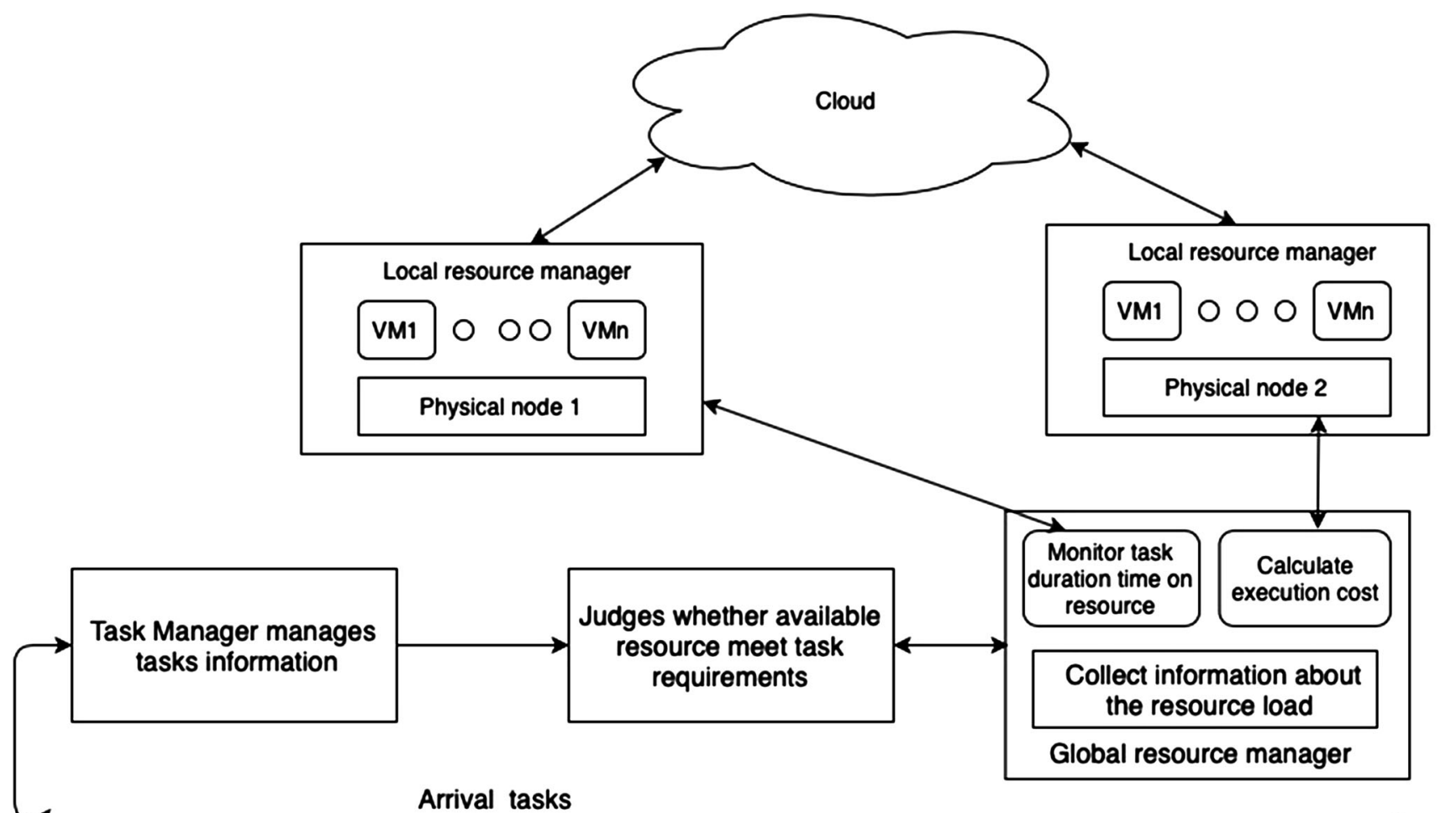

Arrival tasks

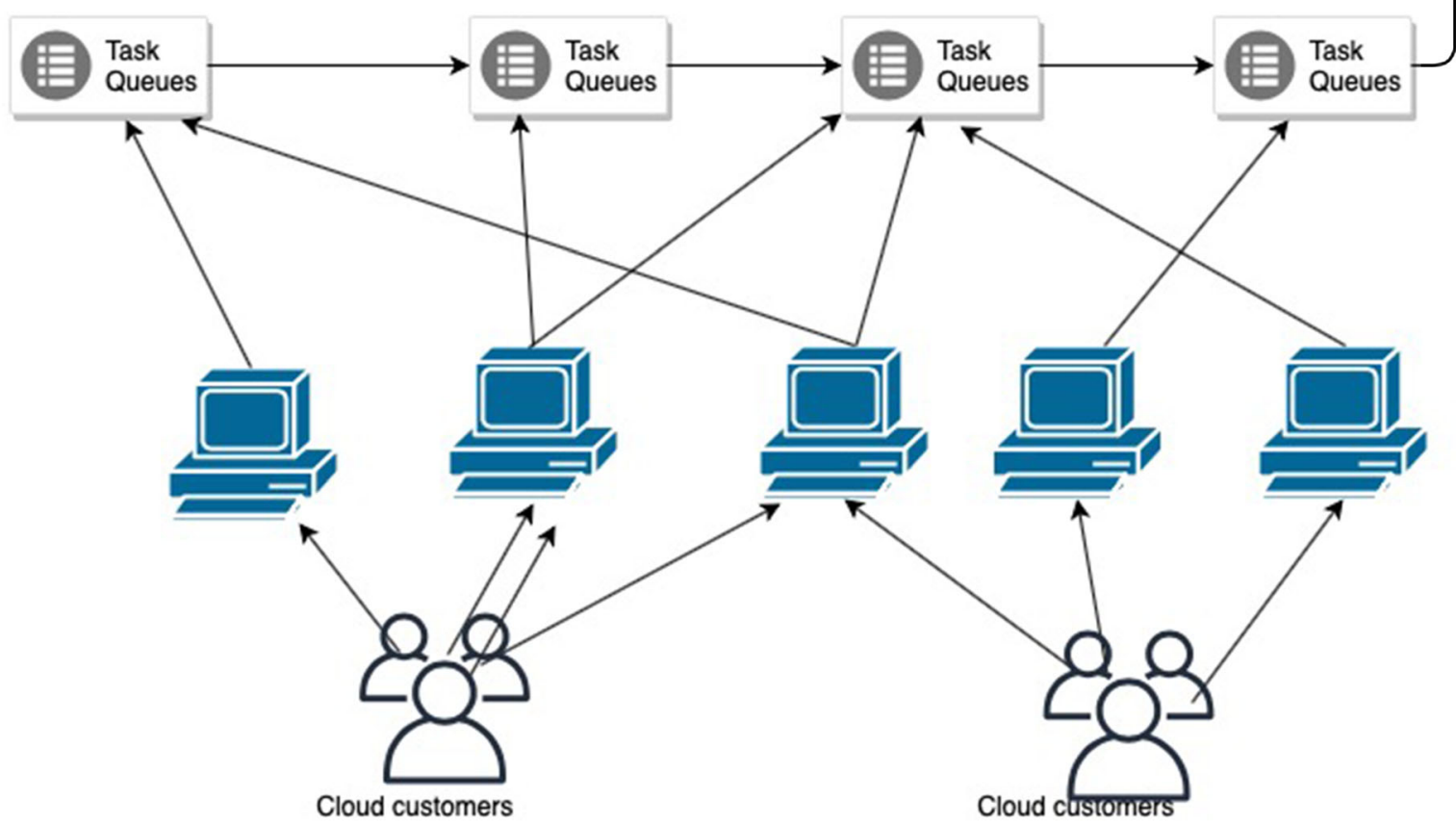

Fig. 1 System model

the relationship between time and cost [14]. A combined method put forward in $[14,15,25]$ was used in the formulation of the multi-objective time-cost model. In our assumption for the formulation of the time and cost model, all virtual machines are said to belong to the same service provider, ignoring the cost of data transfer [39].

\subsubsection{Execution time model}

Let $T=\left\{t_{i} n \geq i \geq 1\right\}$ denote the set of tasks and $V=$ $\left\{v_{k} m \geq k \geq 1\right\}$ the set of heterogeneous virtual machines. Assuming $t_{i} \forall i=\{1,2, \ldots, n\}$ is to be scheduled on $v_{k} \forall k=\{1,2, \ldots, m\}$, the execution time exec $_{k}$ of all tasks 
processed on a virtual machine is computed using Eq. (9) $[15,25]$. The total execution time Texe $_{k}$ of all tasks $t_{i}$ processed on all virtual machines $v_{k}$ is computed using Eq. 10 [25]:

$$
\begin{aligned}
& \operatorname{exec}_{k}=\sum_{i=1}^{n} x_{i k} \cdot \frac{t_{i} v_{k}}{n p e_{k} \cdot v_{k m i p s}} \\
& \text { Texec }_{k}=\sum_{k=1}^{m} \sum_{i=1}^{n} x_{i k} \cdot \frac{t_{i} v_{k}}{n p e_{k} \cdot v_{k m i p s}}
\end{aligned}
$$

where $\operatorname{exec}_{k}$ is the execution time of running tasks on one virtual machine; $x_{i k}$ is equal to 1 if task $t_{i}$ is assigned to a virtual machine, otherwise $x_{i k}=0 ; t_{i}$ is the task whose length is given in million instructions (MIs); $v_{\text {kmips }}$ is the virtual machine speed whose unit is given in million instructions per second (MIPS); and $n p e_{k}$ is the number of processing elements of a virtual machine.

\subsubsection{Execution cost model}

The proposed cost model is a multi-objective task scheduling model that captures the customers' QoS requirement. The model permits charging a customer based on the amount of time the virtual machine spent executing his/or her request [35]. The time quantum [37] of a virtual machine is the smallest discrete unit use by the service providers to define the cost of a virtual machine in either per second or on hourly basis. In this study, we assume the cost of memory and central processing unit (CPU) are all included in the monetary cost of a virtual machine [25]. For instance, assume for every one-minute $N$ of using a virtual machine, the price specified by the service provider is given as 0.5 dollars per hour. For time quantum in minutes of using a virtual machine, the execution cost can be computed as $\frac{N * 0.5}{60}$ dollars [37]. Assuming the cost $v_{k c o s t}$ of executing tasks on a virtual machine per hour (/h) is known, Eq. (11) holds for the execution cost exe $e_{\text {cost }}$ of tasks $t_{i}$ on a virtual machine per time quantum in second [25, 37]:

exe $_{\text {cost }}=\frac{1}{3600} \cdot v_{k c o s t} \cdot \sum_{i=1}^{n} x_{i k} \cdot \frac{t_{i} v_{k}}{n p e_{k} * V_{\text {mips }}}$

where $v_{k c o s t}$ is the monetary cost of a virtual machine per time quantum in US dollar per hour:

$x_{i k}= \begin{cases}1, & \text { if } t_{i} \text { is assign on a virtual machine. } \\ 0, & \text { otherwise }\end{cases}$

When more than one $v_{k} \forall k=\{1,2, \ldots, m\}$ is used by a service provider to execute many tasks, the total tasks execution cost TTexe $_{\text {cost }_{k}}$ by all virtual machine in a datacenter in a datacenter can be computed using Eq. (13):

TTexe $_{\text {cost }_{k}}=\frac{1}{3600} \cdot v_{k c o s t} \cdot \sum_{k=1}^{m} \sum_{i=1}^{n} x_{i k} \cdot \frac{t_{i} v_{k}}{n p e_{k} * V_{\text {mips }_{k}}}$
The multi-objective task scheduling mathematical model can be expressed as follows:

Min $F(X)=\left[\operatorname{Texec}_{k}(X)\right.$, TTexe $\left._{\cos t_{k}}(X)\right]$

subject to

$\sum_{k=1} x_{i k}=1, \quad \forall i=1,2, \ldots, n ; x_{i k} \in\{0,1\}, \forall i, k$

Equation (14) is the proposed multi-objective optimization time-cost model that captured customers' QoS requirement.

\section{The multi-objective scheduling method based on the proposed DMOOTC scheme}

The proposed DMOOTC scheme consists of two phases (global and local search) that are combined to solve the multi-objective task scheduling optimization problem. The following attributes were considered to arrive at an optimal solution: the tasks number, the number of virtual machines and other relevant parameters such as count dimension to change (CDC). Each cat symbolizes the choice of virtual machine used for the task schedule. This is encoded in $\left[\begin{array}{ll}1 \times n \\ \end{array}\right]$ vector, with $n$ belonging to a number of tasks. We also assume that each virtual machine in a datacenter has different cost per time quantum.

Based on expected time to compute (ETC), when tasks are scheduled on a virtual machine by our proposed DMOOTC algorithm, it uses two-level orthogonal array $L_{n}\left(2^{n-1}\right) \forall n \geq N+1$, where $\mathrm{N}$ represents task number. Each task is assigned to a cat (also known as the virtual machine). Each of the cats has a dimension $D$, and the models associated with each cats are based on two objective functions: the total execution time $\left(\operatorname{Texec}_{k}(X)\right)$ and total execution cost $\left(\right.$ TTexe $\left._{\text {cost }_{k}}(X)\right)$. When a cat traverses all tasks, it formed a feasible solution. Each cat has both position and the velocity vector. The position of the cat symbolizes the solution attained by the cat. A mixed ratio (MR) is a control factor that is used to specify two groups of cats. The cats are moved into either seeking or tracing mode at random using the value of the $M R$. When the cat reaches its desired targets, its fitness value is computed based on the defined objective function $\left(\right.$ Texe $_{k}$ and TTexe $_{\text {cost }}$ ). This process of assigning tasks to virtual machine mimics the process of the orthogonal approach. As the velocity of cat points the cat toward achieving near-optimal solution, two sets of candidate velocity vectors $\vec{V}_{\text {set }_{1_{k, d}}}(t)$ and $\vec{V}_{\text {set }_{2 k, d}}(t)$ are generated as follows:

$\left\{\begin{array}{l}\vec{V}_{\text {set }_{k, d}}(t)=w_{1} \cdot \vec{V}_{k, d}(t-1)+\left(c_{1} r_{1} \cdot\left(\vec{X}_{g b s e t_{d}}(t-1)-\vec{X}_{k, d}(t-1)\right)\right. \\ \vec{V}_{\text {set }_{2, d}}(t)=w_{2} \cdot \vec{V}_{k, d}(t-1)+\left(c_{1} r_{1} \cdot\left(\vec{X}_{\text {ibset }_{d}}(t-1)-\vec{X}_{k, d}(t-1)\right)\right.\end{array}\right.$ 
such that:

$\overrightarrow{V o}_{k, d}(t)= \begin{cases}\vec{V}_{\text {set }_{1, d}}(t-1), & \text { if orthogonal array element is " } 1 \text { " } \\ \vec{V}_{\text {set }_{2, d}}(t-1), & \text { otherwise }\end{cases}$

where $\overrightarrow{V o}_{k, d}(t)$ represents two candidate velocity sets; $d$ is dimension of the solution space; $\vec{X}_{\text {gbset }_{d}}$ represents the global best position attained by the cat; $\vec{X}_{\text {lbset }_{d}}$ represents the local best position of the cat; $w_{1}, w_{2}$ are the controlled factors; $r_{1}$ represents uniform random number in the range of $[0,1] ; c_{1}$ represents a constant value of the acceleration;
$\vec{X}_{k, d}$ represents the position of the cat; and $t$, is the number of iterations. To update the velocity, the velocity among the two velocity sets with the best optimum solution is selected using the condition in Eq. (17):

$\vec{V}_{k, d}(t)\left\{\begin{array}{l}\max v, \text { if }\left[\vec{V}_{k, d}(t-1)+\overrightarrow{V o}_{k, d}(t-1)\right]>\text { maximum velocity, } \\ \vec{V}_{k, d}(t-1)+\overrightarrow{V o}_{k, d}(t-1) \quad \text { otherwise }\end{array}\right.$

where $\max v$ is the maximum velocity attained by the cat; $\vec{V}_{k, d}$ represents the velocity attained by the cat; and $\overrightarrow{V o}_{k, d}(t)$ represents the two candidate solutions. A dominant strategy is used to compare the optimum solution and

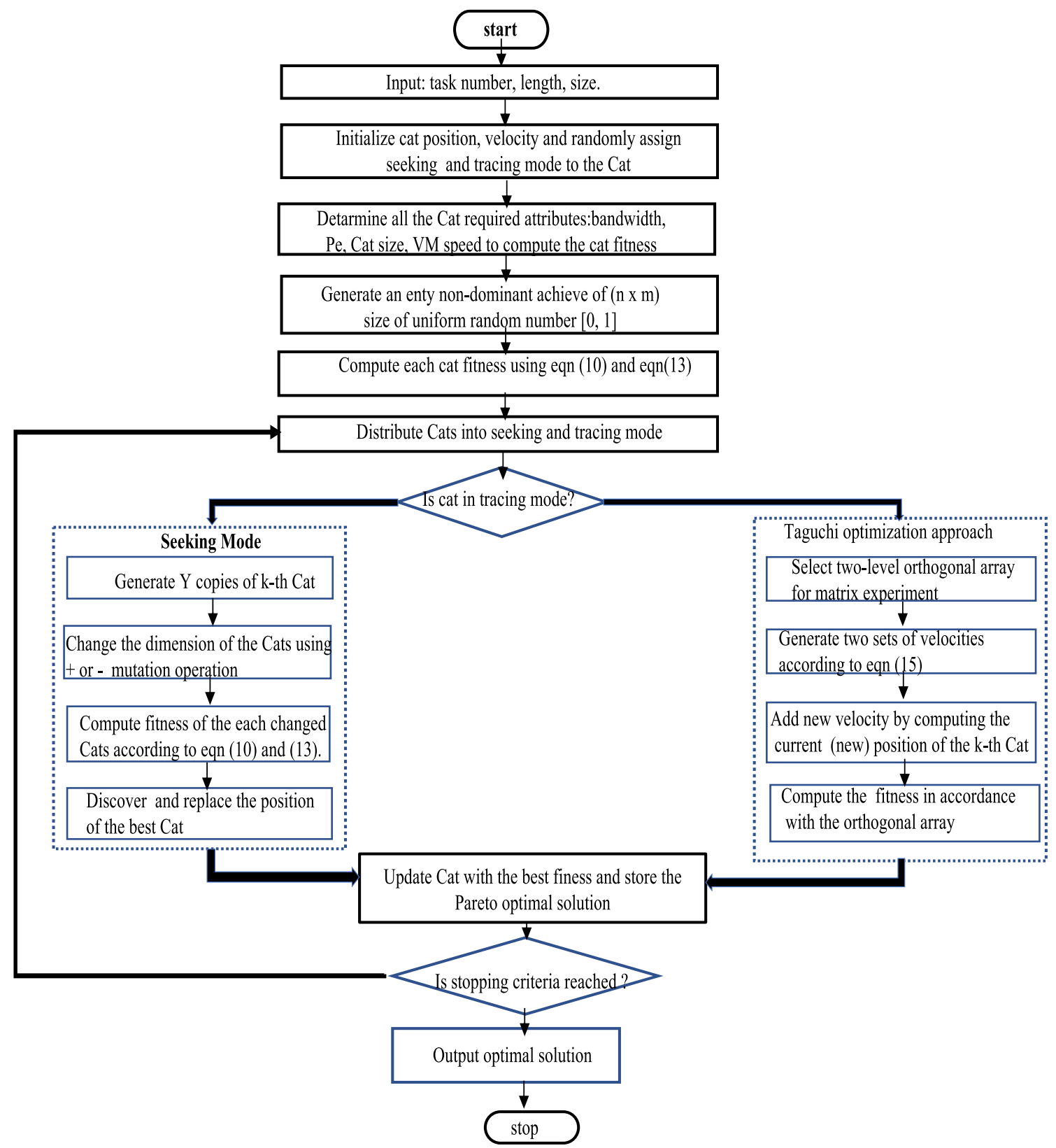

Fig. 2 Flowchart of the DMOOTC scheduling algorithm 
is stored at the archive where the final velocity that should formulate the latest velocity is selected. This velocity returns optimal solution which is used to compute the new position of the cat as indicated in Eq. (18):

$\vec{X}_{k, d}(t)=\vec{X}_{k, d}(t-1)+\vec{V}_{k, d}(t-1)$.

The quality of solution is evaluated using a fitness function. Every cat is assessed based on the value of the fitness function $\operatorname{QoS}(\vec{X})$ in Eq. (19):
$\operatorname{QoS}(\vec{X})=\sum_{j=1}^{m} W_{j} f_{j}\left(\vec{X}_{i}\right),\left\{\forall \overrightarrow{X_{i}} \in\right.$ Archieve $\}$

where $m$ is the number of objective functions and $W_{j}$ is the preference weight for every objective function $\left(f_{j}\left(\vec{X}_{i}\right)\right)$. Algorithm 4 provides the pseudocode for the developed DMOOTC task scheduling algorithm, while Fig. 2 illustrates the flowchart of the scheduling algorithm.

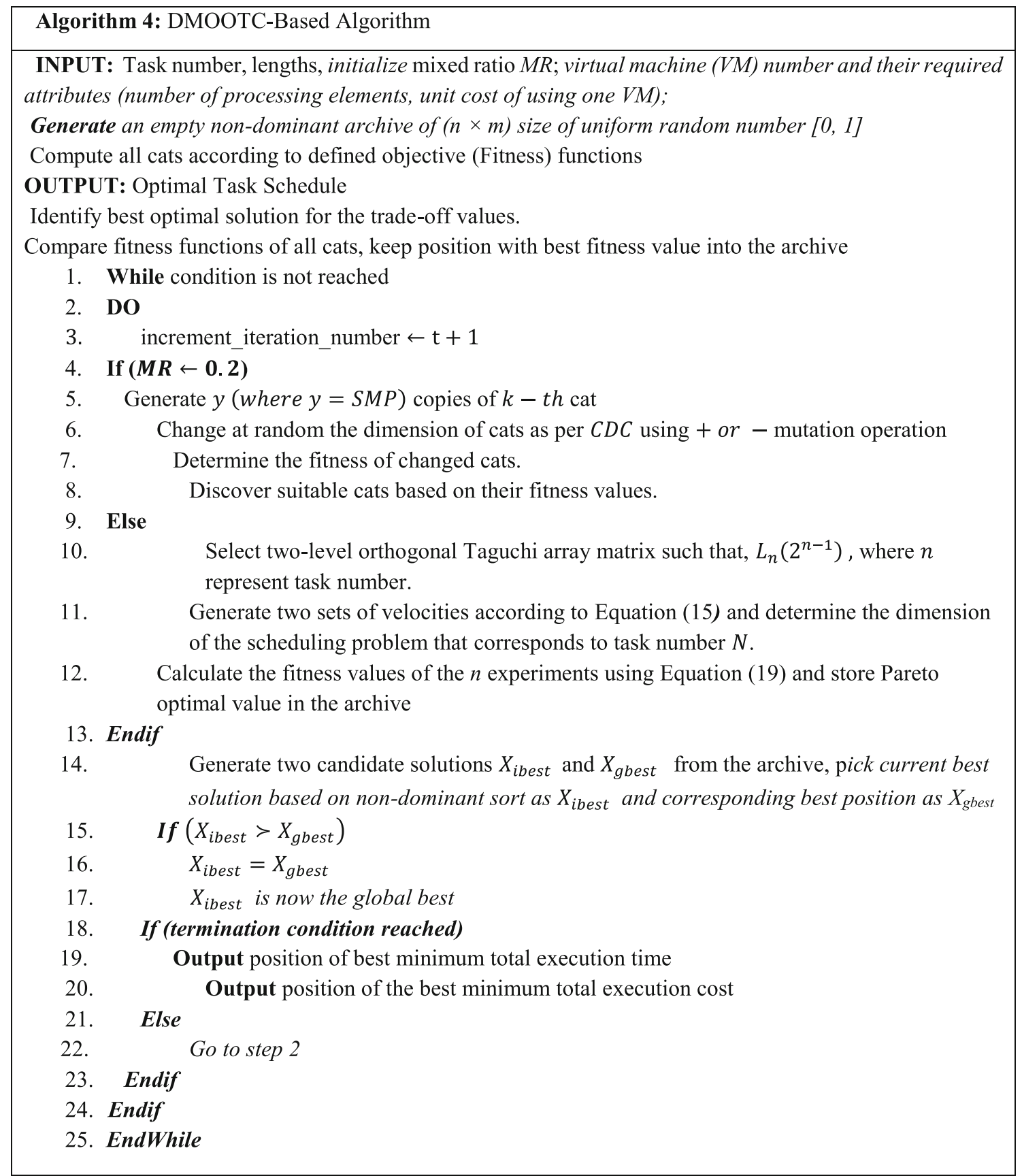


Table 2 Computer and utility software specifications

\begin{tabular}{ll}
\hline Windows specifications & Software \\
\hline Intel $^{\circledR}$ Core $^{\mathrm{TM}}$ i5-5200U CPU @ 3.60 GHz 3.60 GHz & Eclipse-java-luna-SR2-win32-x86-64 \\
Window 10 (64-bit), x64-based processor & CloudSim 3.0.3 [41, 54, 55] \\
4 GB DDR3L RAM & \\
1000 GB(1 TB) SATA-3G HDD & \\
Intel $^{\circledR}$ Core $^{\mathrm{TM}}$ i5-5200U CPU @ 3.60 GHz 3.60 GHz & \\
\hline
\end{tabular}

Table 3 Configured properties for the cloud datacenter

\begin{tabular}{lll}
\hline & Parameter & Values \\
\hline Datacenter & No. of datacenters & 2 \\
& No. of hosts in a datacenter & 1 \\
Host RAM & $2 \mathrm{~GB}$ \\
Storage & ITB \\
Bandwidths & $10 \mathrm{~GB} / \mathrm{s}$ \\
Accumulated host processing power & $1,000,000 \mathrm{MIPS}$ \\
No. of tasks & {$[20-100]$} \\
Lengths & {$[100,1000]$ Mis } \\
File size & {$[200,400] \mathrm{MB}$} \\
Output size & {$[300]$} \\
Number of CPUs & {$[1-4]$} \\
Virtual machine ID & {$[1-20]$} \\
Virtual machines monitor & Xen \\
Accumulated RAM & $0.5 \mathrm{~GB}$ \\
Accumulated storage & $10 \mathrm{~GB}$ \\
Bandwidth & $1 \mathrm{~GB} / \mathrm{s}$ \\
Virtual machine processing power & $1000-10000 \mathrm{MIPS}$ \\
Number of processing elements & 1 to 4 \\
Virtual machine policy & Time-shared \\
Cost per unit of virtual machine & $0.17 \$-1.25 \$ /$ hour \\
Cost of using memory & $0.05 \$ / \mathrm{hour}$ \\
\hline
\end{tabular}

\section{Simulation environment}

Table 2 shows specifications of the computer system and utility software that we used in the simulation of our proposed DMOOTC scheme. The proposed DMOOTC scheme is benchmarked against Multi-Objective Particle Swarm Optimization (MOPSO) [15], Multi-Objective Ant Colony Optimization (MOACO) [24] and Min-Min [40] task scheduling schemes. The selection of properties for the datacenter host, task and virtual machines is used as in $[2,15,40]$. The estimated cost of a unit virtual machine over a time quantum is adopted as used in [24]. This estimated cost comprises both the computing cost and memory cost and varies from one to the other depending on the capacity. The values for the inertia weight and coefficient factors $\left(c_{1} c_{2}\right)$ for MOACO, MOPSO and DMOOTC were specified as used in [59]. Table 3 shows properties of the datacenters, tasks and virtual machines, while Table 4 indicates the parameter settings for the scheduling algorithms.

\section{Performance metrics}

This study considers four performance metrics to evaluate the efficiency of the developed scheme which are discussed in the following:

\subsection{Execution time}

The time a task spent executing on a computing resource (i.e., virtual machine) is significant to cloud customers. To measure the performance of our proposed DMOOTC scheme, the model in Eq. (10) is used. 
Table 4 Parameter settings for the scheduling schemes

\begin{tabular}{lll}
\hline Algorithm & Parameter & Value \\
\hline MOPSO & Particle size & 100 \\
& Self-recognition coefficients $\left(c_{1} c_{2}\right)$ & 2.0 \\
& Uniform random number $\left(R_{1}\right)$ & {$[0,1]$} \\
& Maximum iteration & 1000 \\
& Inertia weight $(W)$ & $90-40 \%$ \\
& Mixed ratio & $2 \%$ \\
& Cat size & 100 \\
DMOOTC & Count dimension to change & $5 \%$ \\
& Cat size & 100 \\
& Self-recognition coefficients $\left(c_{1}\right)$ & 2.0 \\
& Uniform random number $\left(R_{1}\right)$ & {$[0,1]$} \\
& Maximum iteration & 1000 \\
& Inertia weight $(W)$ & $90-40 \%$ \\
& Mixed ratio & $2 \%$ \\
MOACO & Pheromone persistence $\alpha$ & 0.3 \\
& Importance of pheromone $(\gamma)$ & 1 \\
& Importance of resource innate attribute $(\beta)$ & 1 \\
& Pheromone evaporation value $(\rho)$ & 0.3 \\
& Iteration number & 1000 \\
& Number of ant $m$ & 100 \\
\hline \multirow{5}{*}{ MO } &
\end{tabular}

\subsection{Execution cost}

The cost of a service is that a customer paid for the cloud services he/she has consumed [14, 40]. This is derived from the execution cost. In our study, all virtual machines adopted are heterogeneous with varying cost specification per time quantum. Therefore, virtual machines with high speed are said to return high execution cost. Our aim is to provide customers with better choice of virtual machines that will minimize the execution cost using the model developed in Eq. (13).

\subsection{Performance improvement rate}

The performance improvement rate (PIR) is computed in percentage using Eq. (20). It helps to investigate the efficiency of our proposed DMOOTC scheme toward achieving better performance compared to the benchmarked schemes $[2,22]$ :

$\operatorname{PIR}(\%)$

$=\left(\frac{\text { Execution time }(\text { other scheme })-\text { Execution tim }(\text { DMOOTC }))}{\text { Execution time }(\text { other scheme })}\right) \cdot 100$

\subsection{Quality of Service}

The Quality of Service (QoS) represents the fitness of the proposed DMOOTC scheduling scheme based on any combined objective factors. It is used to reveal the quality of standard provided to the customers. The developed algorithm is designed to achieve better QoS [40]. When the execution time and execution cost values are small, QoS value must be higher. In this study, Eq. (19) is adopted in the evaluation of the QoS of the four scheduling schemes $[15,60]$.

\section{Simulation results and discussion}

Two task scheduling scenarios are considered in our experiments. The simulation results are elaborated in these sections. For the first scenario, task instances from 20 to 100 were used with 20 heterogeneous virtual machines, while for the second scenario, High Performance Computing (HPC2N) Net log [61] containing 527, 371 task instances was used. The properties for the datacenters, host and virtual machine settings are similar in configuration to those of the first scenario but vary in terms of the task sizes.

\subsection{First scenario}

For the first scenario, ten independent simulation runs were conducted in revealing the efficiency of our proposed DMOOTC scheme. Tables 5, 6 and 7 show the average value of the simulation runs obtained in terms of execution time and execution cost. Table 5 shows that the Min-Min scheduling scheme was able to achieve better results in the task scheduling interval from 20 to 50. As the scheduling intervals increase over time (see 70 to 100 tasks), performance of the Min-Min decreases to an unprecedented level. On the other hand, the MOACO scheme thus performs better in the task scheduling interval from 20 to 40 . Its performances also degrades with an increase in task sizes above 40 . Weakness in performance recorded by the MOACO scheme is probably caused by the traversing process of the ant colony approach, which usually leads to its entrapment at the local optimal region during the search process. An improvement over MOACO scheme is seen in MOPSO task scheduling scheme. The MOPSO achieves better performance in terms of both the execution time and execution cost under the scheduling interval of 50 to 100 compared to MOACO and Min-Min scheduling schemes.

The performance shown by our proposed DMOOTC scheduling scheme is quite remarkable compared to MinMin, MOACO and MOPSO schemes. Quality-of-Service expectations of customers are paramount to a service 
Table 5 Results of the execution time and cost for the scheduling schemes

\begin{tabular}{|c|c|c|c|c|c|c|c|c|}
\hline \multirow[t]{2}{*}{ Task } & \multicolumn{2}{|c|}{ Improved Min-Min } & \multicolumn{2}{|l|}{ MOACO } & \multicolumn{2}{|l|}{ MOPSO } & \multicolumn{2}{|l|}{ DMOOTC } \\
\hline & $\begin{array}{l}\text { Execution } \\
\text { time (s) }\end{array}$ & $\begin{array}{l}\text { Execution cost } \\
(\$) / \mathrm{h}\end{array}$ & $\begin{array}{l}\text { Execution } \\
\text { time (s) }\end{array}$ & $\begin{array}{l}\text { Execution cost } \\
(\$) / \mathrm{h}\end{array}$ & $\begin{array}{l}\text { Execution } \\
\text { time (s) }\end{array}$ & $\begin{array}{l}\text { Execution cost } \\
(\$) / \mathrm{h}\end{array}$ & $\begin{array}{l}\text { Execution } \\
\text { time }(\mathrm{s})\end{array}$ & $\begin{array}{l}\text { Execution cost } \\
(\$) / \mathrm{h}\end{array}$ \\
\hline 20 & 21.46 & 4.72 & 27.54 & 6.05 & 26.86 & 5.90 & 25.40 & 5.47 \\
\hline 30 & 39.16 & 8.61 & 56.32 & 12.39 & 70.29 & 15.45 & 53.58 & 11.78 \\
\hline 40 & 60.44 & 13.29 & 87.56 & 19.26 & 92.29 & 20.59 & 53.92 & 11.86 \\
\hline 50 & 66.62 & 14.65 & 163.49 & 35.96 & 147.28 & 32.40 & 129.19 & 28.42 \\
\hline 60 & 209.82 & 46.15 & 243.89 & 53.65 & 206.50 & 45.43 & 162.77 & 35.80 \\
\hline 70 & 318.81 & 70.13 & 312.78 & 68.10 & 287.21 & 63.18 & 240.12 & 52.82 \\
\hline 80 & 505.02 & 111.10 & 407.54 & 89.65 & 334.99 & 73.69 & 312.28 & 68.70 \\
\hline 90 & 768.78 & 169.13 & 674.89 & 148.47 & 538.09 & 118.37 & 331.62 & 72.94 \\
\hline 100 & 1096.40 & 241.21 & 754.90 & 166.07 & 663.34 & 145.93 & 454.52 & 99.98 \\
\hline
\end{tabular}

Improved Min-Min Improved Minimum Job First Algorithm, MOACO Multi-Objective Ant Colony Optimization, MOPSO Multi-Objective Particle Swarm Optimization, DMOOTC Dynamic Multi-Objective Orthogonal Taguchi-Cat Algorithm, $h r$ hour

Table 6 Comparison of estimated QoS with cost-time weighted factor $=-0.5$

\begin{tabular}{llrrr}
\hline Task & Improved Min-Min & MOACO & MOPSO & DMOOTC \\
\hline 20 & -10.72 & -13.79 & -13.43 & -12.52 \\
30 & -19.57 & -28.25 & -35.14 & -26.78 \\
40 & -30.22 & -44.01 & -46.80 & -26.96 \\
50 & -33.31 & -82.56 & -73.64 & -64.59 \\
60 & -104.91 & -123.76 & -103.25 & -81.39 \\
70 & -159.53 & -159.34 & -143.61 & -120.06 \\
80 & -252.52 & -208.84 & -269.09 & -156.13 \\
90 & -468.95 & -351.34 & -347.71 & -162.82 \\
100 & -548.25 & -394.86 & -331.68 & -212.10 \\
\hline
\end{tabular}

Improved Min-Min Improved Minimum Job First Algorithm, MOACO Multi-Objective Ant Colony Optimization, MOPSO MultiObjective Particle Swarm Optimization, DMOOTC Dynamic MultiObjective Orthogonal Taguchi-Cat Algorithm

provider for continuous demand of its services. A service provider is expected to meet each customer's expectations within the service-level agreement. In Tables 6 and 7, results of the QoS based on the four scheduling schemes are shown. The weighted factors -0.5 and -0.9 are introduced to serve as a control parameter for customers' service selection in terms of virtual machine types to guarantee minimum execution time and execution cost. Upon successful simulation runs, the total QoS by MinMin, MOACO, MOPSO, and that of the proposed DMOOTC schemes are - 2792.41, - 2497.66, - 2076.19 and -1586.55 . Comparisons show that the proposed DMOOTC scheme has returned the highest QoS value of -1586.55 in contrast to those obtained by the Min-Min, MOACO and MOPSO scheduling schemes. In Table 8
Table 7 Comparison of estimated QoS with time-cost weighted factors $=-0.5$ and -0.9

\begin{tabular}{lcrrr}
\hline Task & Improved Min-Min & MOACO & MOPSO & DMOOTC \\
\hline 20 & -19.31 & -24.80 & -24.17 & -22.54 \\
30 & -35.24 & -50.78 & -63.26 & -48.22 \\
40 & -54.40 & -79.03 & -84.25 & -48.53 \\
50 & -59.96 & -147.95 & -132.55 & -116.27 \\
60 & -188.85 & -220.71 & -185.86 & -146.27 \\
70 & -286.94 & -284.40 & -258.49 & -216.11 \\
80 & -450.93 & -371.86 & -246.30 & -281.06 \\
90 & -709.96 & -621.31 & -484.29 & -298.47 \\
100 & -986.82 & -696.82 & -597.02 & -409.08 \\
\hline
\end{tabular}

Improved Min-Min Improved Minimum Job First Algorithm, MOACO Multi-Objective Ant Colony Optimization, MOPSO MultiObjective Particle Swarm Optimization, DMOOTC Dynamic MultiObjective Orthogonal Taguchi-Cat Algorithm

precisely, the total values for the execution time, execution cost and QoS obtained are reported to provide insight on the performance of the proposed schemes. We further show how significant our proposed DMOOTC scheme in terms of percentage improvement rate as indicated in Table 9. In the overall performance, the proposed DMOOTC scheduling scheme is able to achieve minimum execution time with $42.87 \%, 35.47 \%$ and $25.49 \%$ improvement compared to that of the Min-Min, MOACO and MOPSO task scheduling schemes. In a similar development, DMOOTC scheme is also able achieve $38.62 \%, 35.32 \%$ and $25.56 \%$ improvement compared to the benchmarked schemes. Figures 3 and 4 show the trend of the performances of the scheduling schemes. From the trend 
Table 8 Total QoS obtained by the scheduling schemes

\begin{tabular}{|c|c|c|c|c|}
\hline & Improved Min-Min & MOACO & MOPSO & DMOOTC \\
\hline Total execution time & 3086.51 & 2732.94 & 2366.85 & 1763.40 \\
\hline Total execution cost & 631.84 & 599.60 & 520.94 & 387.77 \\
\hline Total estimated QoS based on cost and time weights $=-0.5$ & -1627.98 & -1406.75 & -1364.35 & -863.35 \\
\hline Total estimated QoS based on cost and time weights $=-0.5$ and -0.9 & -2792.41 & -2497.66 & -2076.19 & -1586.55 \\
\hline
\end{tabular}

Improved Min-Min Improved Minimum Job First Algorithm, MOACO Multi-Objective Ant Colony Optimization, MOPSO Multi-Objective Particle Swarm Optimization, DMOOTC Dynamic Multi-Objective Orthogonal Taguchi-Cat Algorithm

Table 9 Improvement in percentage

\begin{tabular}{lllll}
\hline & Improved Min-Min & MOACO & MOPSO & DMOOTC \\
\hline Total execution time & 3086.51 & 2732.94 & 2366.85 & 1763.40 \\
PIR (\%) over Improved Min-Min & & 11.455 & 23.31 & 42.87 \\
PIR (\%) over MOACO & & & 13.39 & 35.47 \\
PIR (\%) over MOPSO & & & & 25.49 \\
Total execution cost & 631.84 & 599.60 & 520.94 & 387.77 \\
PIR (\%) over Improved Min-Min & & 5.10 & 17.55 & 38.62 \\
PIR (\%) over MOACO & & & 13.11 & 35.32 \\
PIR (\%) over MOPSO & & & & 25.56 \\
\hline
\end{tabular}

Improved Min-Min Improved Minimum Job First Algorithm, MOACO Multi-Objective Ant Colony Optimization, MOPSO Multi-Objective Particle Swarm Optimization, DMOOTC Dynamic Multi-Objective Orthogonal Taguchi-Cat Algorithm, PIR(\%) Performance Improvement Rate (in percentage)

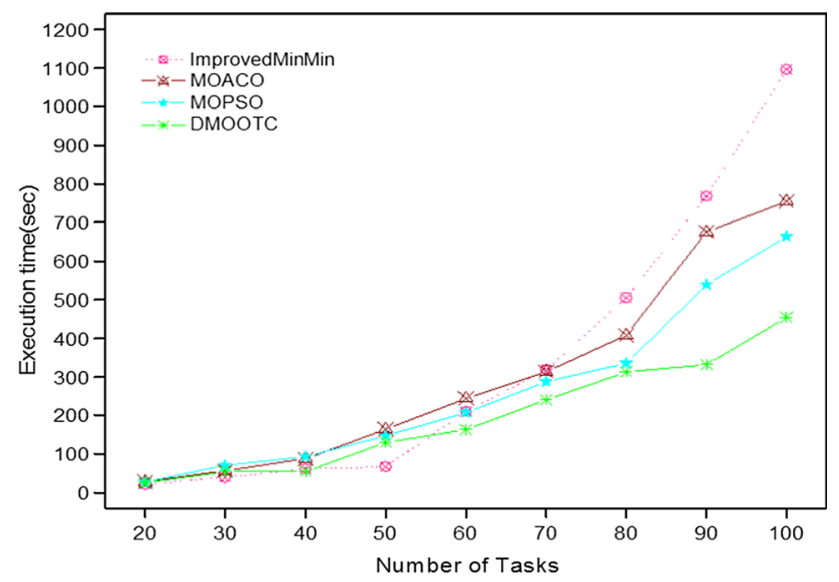

Fig. 3 Total execution time

illustrated in these figures, it has shown that our proposed DMOOTC scheme has the ability to provide customers with the best services in terms of virtual machines that can guarantee them with the minimum execution time and execution cost compared to the benchmarked schemes.

The achievements displayed by our customers service selection scheme are as a result of the incorporation of orthogonal Taguchi at its local search procedure which helps the scheme in traversing all cats (virtual machines), and the use of Pareto dominance strategy provides the best

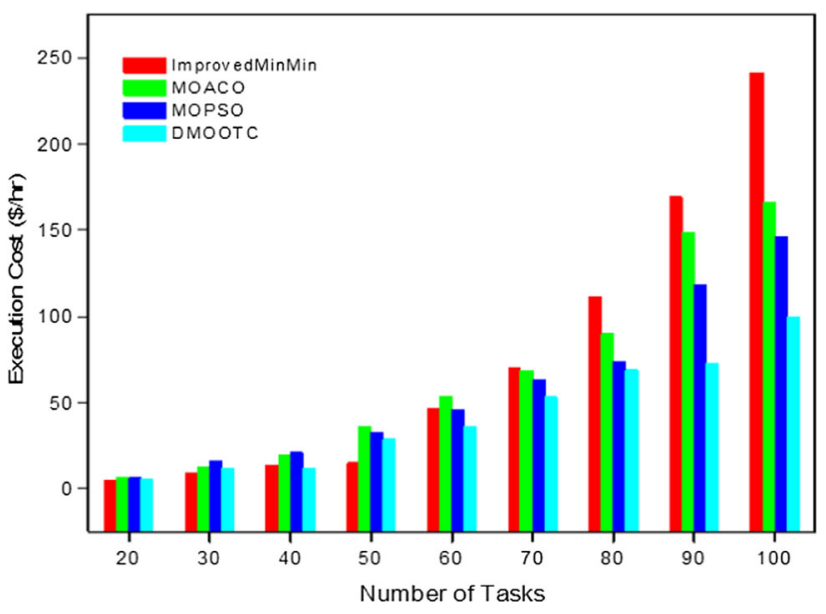

Fig. 4 Total execution cost

optimum solutions of the multi-objective in terms of execution time and execution cost.

\subsection{Second scenario}

In this scenario, we used large-workload-containing HPC2N dataset to determine the performances of the four scheduling schemes. The Parallel Workload ArchiveHPC2N is made available by the High-Performance Computing Center North (HPC2N). It is a setlog with 
Table 10 Results of the execution time and cost for the scheduling schemes

\begin{tabular}{|c|c|c|c|c|c|c|c|c|}
\hline \multirow[t]{2}{*}{ Task } & \multicolumn{2}{|c|}{ Improved Min-Min } & \multicolumn{2}{|l|}{ MOACO } & \multicolumn{2}{|l|}{ MOPSO } & \multicolumn{2}{|l|}{ DMOOTC } \\
\hline & $\begin{array}{l}\text { Execution } \\
\text { time (s) }\end{array}$ & $\begin{array}{l}\text { Execution cost } \\
(\$) / \mathrm{h}\end{array}$ & $\begin{array}{l}\text { Execution } \\
\text { time (s) }\end{array}$ & $\begin{array}{l}\text { Execution cost } \\
(\$) / \mathrm{h}\end{array}$ & $\begin{array}{l}\text { Execution } \\
\text { time (s) }\end{array}$ & $\begin{array}{l}\text { Execution cost } \\
(\$) / \mathrm{h}\end{array}$ & $\begin{array}{l}\text { Execution } \\
\text { time (s) }\end{array}$ & $\begin{array}{l}\text { Execution cost } \\
(\$) / \mathrm{h}\end{array}$ \\
\hline 100 & 486.68 & 107.07 & 967.56 & 212.86 & 1193.18 & 262.50 & 1134.25 & 249.53 \\
\hline 200 & 1452.91 & 319.64 & 1786.67 & 393.06 & 2893.53 & 632.57 & 2564.98 & 564.29 \\
\hline 300 & 3857.67 & 848.68 & 7865.24 & 1730.35 & 6975.66 & 1534.64 & 4587.67 & 1009.28 \\
\hline 400 & 6231.47 & 1370.92 & 9078.56 & 1997.28 & 10411.32 & 2290.49 & 5089.76 & 1119.74 \\
\hline 500 & 9918.75 & 2182.12 & 11436.78 & 2516.09 & 11458.76 & 2520.92 & 8986.08 & 1976.93 \\
\hline 600 & 13810.89 & 3038.39 & 15451.23 & 3399.27 & 12764.87 & 2808.27 & 10679.87 & 2349.57 \\
\hline 700 & 18380.38 & 4043.68 & 16895.87 & 3717.09 & 14023.48 & 3085.16 & 13867.32 & 3050.81 \\
\hline 800 & 22984.71 & 5056.63 & 19417.56 & 4271.86 & 18513.75 & 4073.02 & 16894.90 & 3716.87 \\
\hline 900 & 29688.63 & 6531.48 & 24980.23 & 5495.65 & 23278.90 & 5121.35 & 21856.23 & 4808.37 \\
\hline 1000 & 35424.75 & 7793.44 & 29672.78 & 6528.01 & 26867.23 & 5910.79 & 25785.45 & 5672.79 \\
\hline
\end{tabular}

Improved Min-Min Improved Minimum Job First Algorithm, MOACO Multi-Objective Ant Colony Optimization, MOPSO Multi-Objective Particle Swarm Optimization, DMOOTC Dynamic Multi-Objective Orthogonal Taguchi-Cat Algorithm, $h r$ hour

Table 11 Estimated QoS with time-cost weight factor $=-0.5$

\begin{tabular}{lcrrr}
\hline Task & Improved Min-Min & MOACO & \multicolumn{1}{c}{ MOPSO } & DMOOTC \\
\hline 100 & -243.35 & -483.80 & -596.62 & -567.15 \\
200 & -726.50 & -893.38 & -1446.85 & -1282.56 \\
300 & -1928.95 & -3932.86 & -3488.04 & -2293.97 \\
400 & -3115.92 & -4539.55 & -5205.97 & -2545.03 \\
500 & -4959.67 & -5718.73 & -5729.73 & -4493.31 \\
600 & -6905.86 & -7726.08 & -6382.82 & -5340.26 \\
700 & -9190.75 & -8448.45 & -7012.16 & -6934.08 \\
800 & -11493.05 & -9709.37 & -10183.12 & -8447.96 \\
900 & -14845.22 & -12490.87 & -11640.16 & -10928.78 \\
1000 & -17713.45 & -14837.29 & -13434.43 & -12893.51 \\
\hline
\end{tabular}

Improved Min-Min Improved Minimum Job First Algorithm, MOACO Multi-Objective Ant Colony Optimization, MOPSO Multi-Objective Particle Swarm Optimization, DMOOTC Dynamic Multi-Objective Orthogonal Taguchi-Cat Algorithm

\begin{tabular}{lcrrr}
\hline Task & Improved Min-Min & MOACO & MOPSO & DMOOTC \\
\hline 100 & -438.03 & -870.83 & -1073.90 & -1020.85 \\
200 & -1307.66 & -1608.05 & -2604.27 & -2308.56 \\
300 & -3472.02 & -7078.95 & -6278.31 & -4129.04 \\
400 & -5608.51 & -8170.98 & -9370.50 & -4580.93 \\
500 & -8927.17 & -10293.45 & -10313.23 & -8087.74 \\
600 & -12930.22 & -13906.57 & -11488.77 & -9612.20 \\
700 & -16542.90 & -15206.79 & -12621.56 & -12481.01 \\
800 & -20686.94 & -17476.39 & -16662.94 & -15205.92 \\
900 & -26720.67 & -22482.97 & -20951.72 & -19671.27 \\
1000 & -31883.35 & -26706.40 & -24181.32 & -23207.69
\end{tabular}

Improved Min-Min Improved Minimum Job First Algorithm, MOACO Multi-Objective Ant Colony Optimization, MOPSO Multi-Objective Particle Swarm Optimization, DMOOTC Dynamic Multi-Objective Orthogonal Taguchi-Cat Algorithm 
Table 13 Total QoS obtained by the scheduling schemes

\begin{tabular}{|c|c|c|c|c|}
\hline & Improved Min-Min & MOACO & MOPSO & DMOOTC \\
\hline Total execution time & $142,236.84$ & $137,552.48$ & $128,380.68$ & $111,446.51$ \\
\hline Total execution cost & $31,292.05$ & $30,261.52$ & $28,239.71$ & $24,518.18$ \\
\hline Total estimated QoS based on cost and time weights $=-0.5$ & $-71,122.72$ & $-68,780.38$ & $-65,119.90$ & $-48,792.35$ \\
\hline Total estimated QoS based on cost and time weights $=-0.5$ and -0.9 & $-128,517.47$ & $-123,801.38$ & $-115,546.52$ & $-100,305.31$ \\
\hline
\end{tabular}

Improved Min-Min Improved Minimum Job First Algorithm, MOACO Multi-Objective Ant Colony Optimization, MOPSO Multi-Objective Particle Swarm Optimization, DMOOTC Dynamic Multi-Objective Orthogonal Taguchi-Cat Algorithm

Table 14 Improvement in percentage

\begin{tabular}{lllll}
\hline & Improved Min-Min & MOACO & MOPSO & DMOOTC \\
\hline Total execution time & $142,236.84$ & $137,552.48$ & $128,380.68$ & $111,446.51$ \\
PIR (\%) over Improved Min-Min & & 3.29 & 9.74 & 21.64 \\
PIR (\%) over MOACO & & 6.66 & 18.97 \\
PIR (\%) over MOPSO & & & 13.19 \\
Total execution cost & $31,292.05$ & $30,261.52$ & $28,239.71$ & $24,518.18$ \\
PIR (\%) over Improved Min-Min & & 3.29 & 9.75 & 21.64 \\
PIR (\%) over MOACO & & 6.66 & 18.97 \\
PIR (\%) over MOPSO & & & 13.19 \\
\hline
\end{tabular}

Improved Min-Min Improved Minimum Job First Algorithm, MOACO Multi-Objective Ant Colony Optimization, MOPSO Multi-Objective Particle Swarm Optimization, DMOOTC Dynamic Multi-Objective Orthogonal Taguchi-Cat Algorithm, PIR(\%) Performance Improvement Rate (in percentage) information on about 527, 371 tasks. The HPC2N workload log was freely provided by Ake Sandgren, who also helped with background information and interpretation, while Michael Jack assisted in making sure the log is hosted into the archive for general usage [2]. We stored the workload in a sac folder at the CloudSim. The datacenter broker within the CloudSim is configured to make use of the scheduling schemes as the main scheduler instead of using the default scheduler. Tasks are pooled from the sac folder containing the HPC2N dataset for each run of the simulation based on assigned scheduling intervals (100-1000) using each of the scheme sets for the simulation.

The results after simulations are indicated in Tables 10, $11,12,13$ and 14. These results attest to the fact that our proposed DMOOTC scheme can provide minimum execution time and execution cost compared to improved Min-Min, MOACO and MOPSO schemes. More precisely, Table 13 summarizes the whole results in terms of total execution time and cost. The proposed DMOOTC scheduling scheme is able to achieve - 48792.35 QoS value when a customer selects -0.5 weight factor for his/ or her cloud service and also achieves - 1000305.31 for time and cost weight factors of -0.5 and -0.9 . Table 14 reports a significant improvement gained by our proposed DMOOTC scheme over improved Min-Min, MOACO and MOPSO scheduling schemes in terms of execution time and execution cost. The proposed DMOOTC scheme has

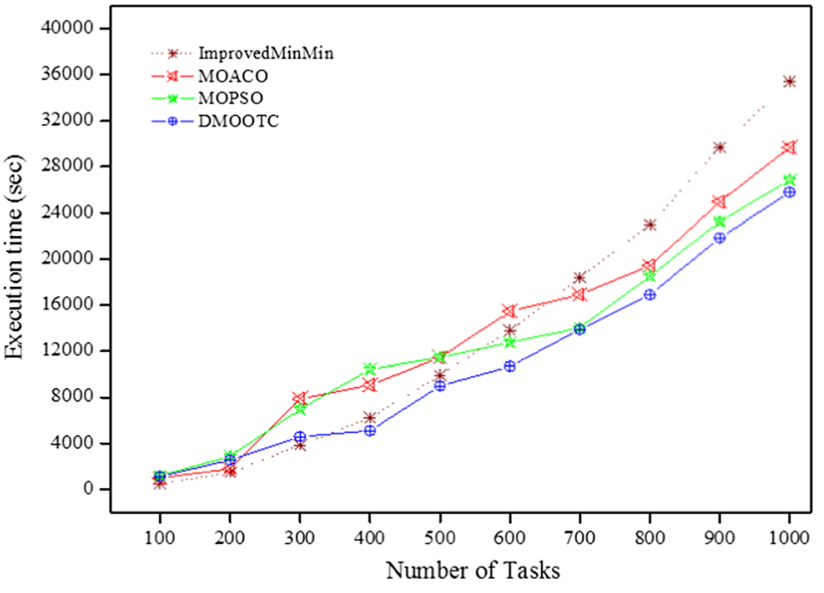

Fig. 5 Total execution time

achieved $21.64 \%, 18.97 \%$ and $13.17 \%$ improvement compared to improved Min-Min, MOACO and MOPSO algorithms. The continuous display of performance by our proposed DMOOTC scheduling scheme is attributed to the incorporation of orthogonal Taguchi approach and the use of Pareto dominance strategy to provide customers with the choices of virtual machines that help in retuning an optimum solution of the multi-objective problem. Likewise, figures are used to further show the trend in performance of the scheduling scheme under different scheduling intervals as shown in Figs. 5 and 6. 


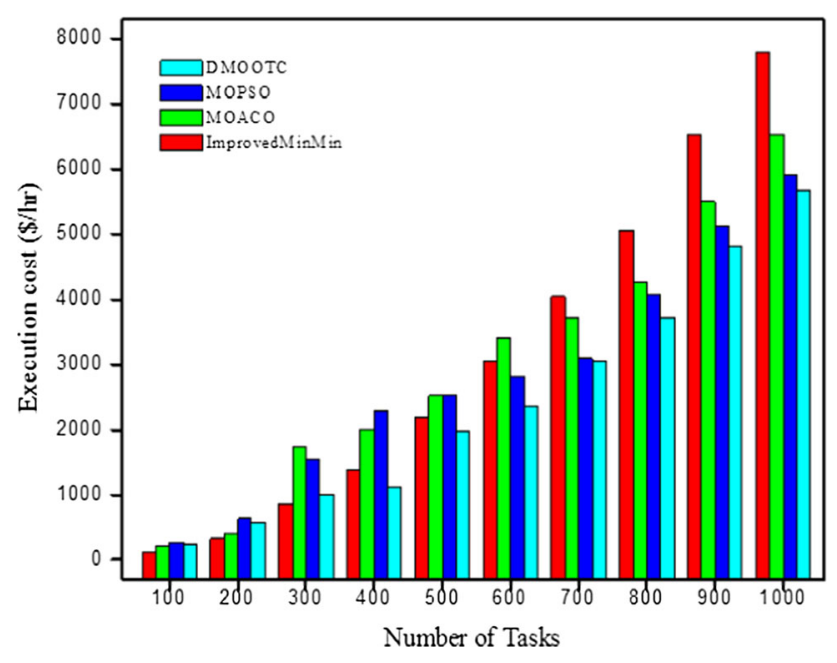

Fig. 6 Total execution cost

\section{Statistical analysis on $95 \%$ confidential interval}

A statistical analysis based on $95 \%$ confidence interval is provided to show how significant our obtained results for both scenarios compared to those of the benchmarked schemes. Tables 15 and 16 present the computed 95\% confidence intervals for both scenarios according to Eq. (21) [62]:

Confidential Interval $(\mathrm{CI})=\bar{x} \pm t \frac{s}{\sqrt{n}}$

where $\bar{x}$ represents the mean; $t$ represents t-distribution that is derived from the t-distribution table; $s$ represents the standard deviation of the sample data and $n$ represents the number of samples. The smaller the value of the confidence interval, the more precise our estimate. For results shown in Tables 15 and 16, the 95\% confidence intervals obtained by our proposed DMOOTC scheduling scheme are less compared to those obtained by the benchmarked schemes. This means that there is a significant difference in the results obtained by our proposed DMOOTC scheduling schemes compared to the benchmarked schemes. It can be concluded that our proposed DMOOTC scheduling scheme can provide cloud customers with better services that will meet their expectations and adapt the elasticity of cloud computing environment than the benchmarked scheduling schemes.
Table 15 Computed $95 \%$ confidence interval for the first scenario

\begin{tabular}{lllll}
\hline & Improved Min-Min & MOACO & MOPSO & DMOOTC \\
\hline Degree of freedom & 9 & 9 & 9 & 9 \\
Confidence level & 0.025 & 0.025 & 0.025 & 0.025 \\
$t$-distribution & 2.262 & 2.267 & 2.267 & 2.262 \\
Mean & 342.94 & 303.21 & 262.98 & 195.93 \\
Standard deviation & 378.38 & 264.49 & 218.04 & 148.25 \\
Lower bound & 72.28 & 114.02 & 107.01 & 90.04 \\
Upper bound & 613.60 & 492.40 & 418.95 & 254.14 \\
95\% confidential interval & $(72.28,613.60)$ & $(114.02,492.40)$ & $(107.01,418.95)$ & $(90.04,254.14)$ \\
\hline
\end{tabular}

Table 16 Computed $95 \%$ confidence interval for the second scenario

\begin{tabular}{lllll}
\hline & Improved Min-Min & MOACO & MOPSO & DMOOTC \\
\hline Degree of freedom & 9 & 9 & 9 & 9 \\
Confidence level & 0.025 & 0.025 & 0.025 & 0.025 \\
$t$-distribution & 2.262 & 2.267 & 2.267 & 2.262 \\
Mean & $11,868.01$ & 11986.63 & 11279.27 & 9517.90 \\
Standard deviation & $10,155.57$ & 7995.00 & 7048.25 & 6979.87 \\
Lower bound & 3378.74 & 3412.51 & 3211.13 & 2709.68 \\
Upper bound & $20,357.28$ & $20,560.75$ & $19,347.41$ & $16,326.12$ \\
95\% confidential interval & $(3378.74,20,357.28)$ & $(3412.51,20,560.75)$ & $(3211.13,19,347.41)$ & $(2709.68,16,326.12)$ \\
\hline
\end{tabular}




\section{Conclusion}

Scheduling of cloud service for the purpose of meeting the expectations of each customer in cloud computing is a nondeterministic polynomial times (NP-hard) hard problem. Solutions required to facilitate the provisioning of better cloud service are rather too complex to develop. In this paper, we proposed a cloud customers service selection scheme known as Dynamic Multi-Objective Orthogonal Taguchi-Cat (DMOOTC) that served as an ideal solution. The proposed DMOOTC scheduling scheme not only considers meeting customers' QoS expectations, but also facilitates the provisioning of several service choices for customers to select their service preference. Two computing scenarios were adopted in evaluation of the efficiency of our proposed DMOOTC scheduling scheme via simulation. The simulation results obtained in both scenarios show our proposed DMOOTC scheduling scheme had returned minimum execution time and cost for all scheduled tasks and also provided better QoS compared to the benchmarked schemes. We further revealed the significance of our proposed DMOOTC scheduling scheme using statistical analysis based on $95 \%$ confidence interval. The statistical results obtained by our proposed DMOOTC scheduling scheme are quite significant than those obtained by the benchmarked schemes. The overall performances displayed by our proposed DMOOTC scheme is as a result of the incorporation of an orthogonal Taguchi strategy at its local search, which facilitates better task mapping on virtual machines and the use of Pareto dominance strategy that provides customers with several service choices to select their preference. Further studies are therefore necessary to investigate the scalability of the proposed DMOOTC scheduling scheme using large workloads.

Acknowledgements Open access funding provided by Umea University. The use of Universiti Teknologi Malaysia facilities and its services is hereby acknowledged with thanks.

\section{Compliance with ethical standards}

Conflict of interest There is no conflict of interest for this manuscript. All authors agreed to its submission.

Open Access This article is licensed under a Creative Commons Attribution 4.0 International License, which permits use, sharing, adaptation, distribution and reproduction in any medium or format, as long as you give appropriate credit to the original author(s) and the source, provide a link to the Creative Commons licence, and indicate if changes were made. The images or other third party material in this article are included in the article's Creative Commons licence, unless indicated otherwise in a credit line to the material. If material is not included in the article's Creative Commons licence and your intended use is not permitted by statutory regulation or exceeds the permitted use, you will need to obtain permission directly from the copyright holder. To view a copy of this licence, visit http://creativecommons. org/licenses/by/4.0/.

\section{References}

1. Gui Z, Yang C, Xia J, Huang Q, Liu K, Li Z, Yu M et al (2016) A service brokering and recommendation mechanism for better selecting cloud services. PLoS ONE 9(8):e105297. https://doi. org/10.1371/journal.pone.0105297

2. Abdullahi M, Ngadi MA, Abdulhamid SM (2016) Symbiotic Organism Search optimization-based task scheduling in cloud computing environment. Future Gener Comput Syst 56(2016):640-650

3. Thanasias V, Lee C, Hanif M, Kim E, Helal S (2016) VM capacity-aware scheduling within budget constraints in IaaS clouds. PLoS ONE 11(8):e0160456. https://doi.org/10.1371/jour nal.pone.0160456

4. Gabi D (2014) Surveillance on security issues in cloud computing: a view on forensic perspective. Int J Sci Eng Res 5(5): 1246-1252

5. Gabi D, Ismail AS, Zainal A (2015) Systematic review on existing load balancing techniques in Cloud Computing. Int $\mathbf{J}$ Comput Appl 125(9):16-24

6. Meena M., Bharadi VA (2016). Performance analysis of cloud based software as a service (SaaS) model on public and hybrid cloud. In: Proceedings of the 2016 symposium on colossal data analysis and networking (CDAN). 18-19 March. Indore, Madhya Pradesh, India, pp 1-6

7. Tassabehji R, Hackney R (2016) Hey You? Get Off My Cloud: evaluation of cloud Service Models for Business Value within Pharma X. J Adv Manag Sci Inf Syst 2:48-52

8. Zhang Y, Qian C, Lv J, Liu Y (2017) Agent and cyber-physical system based self-organizing and self-adaptive intelligent shopfloor. IEEE Trans Ind Inf 13(2):737-747

9. Furht B (2010) Cloud computing fundamentals. In: Furht B, Escalante A (eds) Handbook of cloud computing. Springer, New York, pp 3-19

10. Raza HM, Adenola FA, Nafarieh A, Robertson W (2015) The slow adoption of cloud computing and it workforce. Proc Comput Sci 52(2015):1114-1119

11. Zhan Z-H, Liu X-F, Gong Y-J, Zhang J, Chung HS-H, Li Y (2015) Cloud computing resource scheduling and a survey of its evolutionary approaches. J ACM Comput Survey 47(4):1-33

12. Cui H, Liu X, Yu T, Zhang H, Fang Y, Xia Z (2017) Cloud service scheduling algorithm research and optimization. Hindawi Secur Commun Netw 2017:1-7

13. Gabi D, Ismail AS, Zainal A, Zakaria Z (2018) Quality of Service (QoS) task scheduling algorithm with taguchi orthogonal approach for cloud computing environment. In: Saeed F, Gazem N, Patnaik S, Saed Balaid A, Mohammed F (eds) Recent Trends in Information and Communication Technology. IRICT 2017. Lecture notes on data engineering and communications technologies, vol 5. Springer, Cham, pp 641-649

14. Zuo L, Shu L, Dong S, Zhu C, Hara T (2015) A multi-objective optimization scheduling method based on the ant colony algorithm in cloud computing. IEEE Access J Rapid Open Access Publ 3:2687-2699

15. Ramezani F, Lu J, Taheri J, Hussain FK (2013). Task scheduling optimization in cloud computing applying multi-objective particle swarm. LNCS 8274, 2013: Springer, Berlin, pp 237-251

16. Zhang F, Cao J, Li K, Khan US, Hwang K (2014) Multi-objective scheduling of many tasks in cloud platforms. Futur Gener Comput Syst 37(2014):309-320

17. Xie Z, Shao X, Xin Y (2016) A scheduling algorithm for cloud computing system based on the driver of dynamic essential path. PLoS ONE 11(8):e0159932. https://doi.org/10.1371/journal.pone. 0159932 
18. Deb K (2001) Multi-objective optimization using evolutionary algorithms. Wiley, New York

19. Subashini G, Bhuvaneswari MC (2011) Non dominant particle swarm optimization for scheduling independent tasks on heterogeneous distributed environments. Int $\mathbf{J}$ Adv Soft Comput Appl 3(1): $1-17$

20. Gabi D, Ismail AS, Zainal Zakaria Z (2017) Solving task scheduling problem in cloud computing environment using orthogonal Taguchi-cat algorithm. Int J Electr Comput Eng (IJECE) 7(3):1489-1497

21. Souza Pardo MH, Centurion AM, Franco Eustáquio PS, Carlucci Santana RH, Bruschi SM, Santana MJ (2016) Evaluating the influence of the client behavior in cloud computing. PLoS ONE 11(7):e0158291. https://doi.org/10.1371/journal.pone.0158291

22. Abdulhamid SM, Abd Latiff MS, Abdul-Salaam G, Hussain Madni SH (2016) Secure scientific applications scheduling technique for cloud computing environment using global league championship algorithm. PLoS ONE 11(7):e0158102. https://doi. org/10.1371/journal.pone.0158102

23. Tran D-H, Cheng M-Y, Cao M-T (2015) Hybrid multiple-objective artificial bee colony with differential evolution for the time-cost-quality tradeoff problem. Knowl Based Syst 74(2015):176-186

24. Lakra AV, Yadav DK (2015) Multi-objective task scheduling algorithm for cloud computing throughput optimization. Proc Comput Sci J 48(2015):107-113

25. Ramezani F, Lu J, Taheri J, Hussain FK (2015) Evolutionary algorithm-based multi-objective task scheduling optimization model in cloud environments. World Wide Web 18:1737-1757. https://doi.org/10.1007/s11280-015-0335-3

26. Rubio JJ, Lughofer E, Meda-Campaña JA, Páramo LA, Novoa JF, Pacheco J (2018) Neural network updating via argument Kalman filter for modeling of Takagi-Sugeno fuzzy models. J Intell Fuzzy Syst 35(2):2585-2596

27. Soares AM, Fernandes BJT, Bastos-Filho CJA (2018) Pyramidal neural networks with evolved variable receptive fields. Neural Comput Appl 29(12):1443-1453

28. Rubio JJ (2017) USNFIS: uniform stable neuro fuzzy inference system. Neurocomputing 262:57-66

29. Liu Y, Wang Z, Yuan Y, Alsaadi FE (2018) Partial-nodes-based state estimation for complex networks with unbounded distributed delays. IEEE Trans Neural Netw Learn Syst 29(8):3906-3912

30. Rubio JJ (2009) SOFMLS: online self-organizing fuzzy modified least-squares network. IEEE Trans Fuzzy Syst 17(6):1296-1309

31. Li X, Li H, Sun B, Wang F (2018) Assessing information security risk for an evolving smart city based on fuzzy and grey FMEA. J Intell Fuzzy Syst 34(4):2491-2501

32. Chu S-C, Tsai P-W (2007) Computational intelligence based on the behavior of cats. Int $\mathrm{J}$ Innov Comput Inf Control 3(2007):163-173

33. Gabi D, Ismail AS, Zainal A, Zakaria Z, Abraham A (2016) Orthogonal Taguchi-based cat algorithm for solving task scheduling problem in cloud computing. Neural Comput Appl (2016) 30(6):1845-1863

34. Panda SK, Jana PK (2015) A Multi-objective task scheduling algorithm for heterogeneous multi-cloud environment. In: Proceedings of the international conference on electronic design, computer networks and automated verification (EDCAV), 2015. IEEE

35. Pacini E, Mateos C, Garino CG (2015) Balancing throughput and response time in online scientific Clouds via Ant Colony Optimization. Adv Eng Softw 84:31-47

36. Wei X, Sun B, Cui J, Xu G (2016) A multi-objective compounded local mobile cloud architecture using priority queues to process multiple jobs. PLoS ONE 11(7):e0158491. https://doi. org/10.1371/journal.pone.0158491

37. Liu J, Pacitti E, Valduriez P, de Oliveira D, Mattoso M (2016) Multi-objective scheduling of Scientific Workflows in multisite clouds. Futur Gener Comput Syst 63(2016):76-95

38. Duan R, Prodan R, Li X (2014) Multi-objective game theoretic scheduling of bag-of-tasks workflows on hybrid clouds. IEEE Trans Cloud Comput 1 2:29-42

39. Verma A, Kaushal S (2015) Cost-time efficient scheduling plan for executing workflows in the cloud. J Grid Comput 2015:495-506. https://doi.org/10.1007/s10723-015-9344-9

40. Liu G, Li J, Xu J (2013) An improved min-min algorithm in cloud computing. Adv Intell Syst Comput. https://doi.org/10. 1007/978-3-642-33030-8_8

41. Beegom ASA, Rajasree MS (2014) A particle swarm optimization based pareto optimal task scheduling in cloud computing. Lect Notes Comput 8795:79-86

42. Akbari M, Rashidi H (2016) A multi-objectives scheduling algorithm based on cuckoo optimization for task allocation problem at compile time in heterogeneous systems. Expert Syst Appl 60(2016):234-248

43. Voicu C, Pop F, Dobre C, Xhafa F (2014) MOMC: multi-objective and multi-constrained scheduling algorithm of many tasks in Hadoop. In: Proceedings of the ninth international conference on P2P, parallel, grid, cloud and internet computing (3PGCIC); 2014: IEEE

44. Bilgaiyan S, Sagnika S, Das M (2015) A multi-objective cat swarm optimization algorithm for workflow scheduling in cloud computing environment. Int J Soft Comput 10(1):37-45

45. Xu Z, Xu X, Zhao X (2015) Task scheduling based on multiobjective genetic algorithm in cloud computing. J Inf Comput Sci 12(4):1429-1438

46. Milani FS, Navin AH (2015) Multi-objective task scheduling in the cloud computing based on the patrice swarm optimization. Int J Inf Technol Comput Sci 7(5):61-66

47. Jena RK (2015) Multi-objective task scheduling in cloud environment using nested PSO framework. Proc Comput Sci J 57(2015):1219-1227

48. Khajehvand V, Pedram H, Zandieh M (2014) Multi-objective and scalable heuristic algorithm for workflow task scheduling in utility grids. J Optim Ind Eng 14(2014):27-36

49. Pradhan PM, Panda G (2012) Solving Multi-objective problems using cat swarm optimization. Int $\mathrm{J}$ Expert Syst Appl 39(2012):2956-2964

50. Shojaee R, Faragardi R. H, Alaee S, Yazdani N (2012) A New cat swarm optimization based algorithm for reliability-oriented task allocation in distributed systems. In: symposium on sixth international telecommunications (IST). IEEE

51. Taguchi G, Chowdhury S, Taguchi S (2000) Robust engineering: learn how to boost quality while reducing costs and time to market. McGraw-Hill, New York

52. Tsai P-W, Pan J-S, Chen S-M, Lio B-Y (2012) Enhanced parallel cat swarm optimization based on taguchi method. Expert Syst Appl 39(2012):6309-6319

53. Tsai J-T, Fang J-C, Chou J-H (2013) Optimized tasks scheduling and resource allocation on cloud computing environment using improved differential evolution algorithm. Comput Oper Res 40(2013):3045-3055

54. Chang H-C, Chen Y-P, Liu T-K, Chou J-H (2015) Solving the flexible job shop scheduling problem with makespan optimization by using a hybrid Taguchi-genetic algorithm. IEEE J Mag 3:1740-1754

55. Tsai J-T, Liu T-K, Ho W-H, Chou J-H (2008) An improved genetic algorithm for job-shop scheduling problems using Taguchi-based crossover. Int $\mathrm{J}$ Adv Manuf Technol 2008(38):987-994 
56. Saule C, Giegerich R (2015) Pareto optimization in algebraic dynamic programming. Algorithms Mol Biol 10(22):1-20

57. Kalra M, Singh S (2015) A review of metaheuristic scheduling techniques in cloud computing. Egypt Inf J 16(3):275-295

58. Farahabady HRM, Lee CY, Zomaya YA (2014) Pareto-optimal cloud bursting. IEEE Trans Parallel Distrib Syst 25(10):2670-2682

59. Eberhart RC, Shi Y (2000) Comparing inertia weights and constriction factors in particle swarm optimization. In: Proceedings of the IEEE conference on evolutionary computation (ICEC). IEEE
60. Calheiros RN, Ranjan R, Beloglazov A, De Rose CAF, Buyya R (2010) CloudSim: a toolkit for modeling and simulation of cloud computing environments and evaluation of resource provisioning algorithms. Softw Pract Exp 41(1):23-50

61. HPC2N.TheHPC2NSethlog (2016) http://www.cs.huji.ac.il/labs/ parallel/workload/1_hpc2n/index.html. Accssed 12 Apr 2018

62. Hosmer DW, Lemeshow S (1992) Confidence interval estimation of interaction. Epidemiology 3(5):452-456

Publisher's Note Springer Nature remains neutral with regard to jurisdictional claims in published maps and institutional affiliations. 